\title{
Systemic inflammation causes microglial dysfunction with a mixed AD-like pathology.
}

Praveen Bathini ${ }^{1}$, Isabel Dupanloup ${ }^{2}$, Elena Zenaro ${ }^{3}$, Eleonora Terrabuio ${ }^{3}$, Amrei Fischer ${ }^{1}$, Edona Ballabani ${ }^{1}$, Marie-Agnes Doucey ${ }^{4}$, Lavinia Alberi ${ }^{1,5}$

${ }^{1}$ Department of Medicine, University of Fribourg, Fribourg, Switzerland

2 Swiss Institute for Bioinformatics, Lausanne, Switzerland

${ }^{3}$ Department of Medicine, Section of General Pathology, University of Verona, Verona, Italy

${ }^{4}$ University of Lausanne, Lausanne, Switzerland

${ }^{5}$ Swiss Integrative Center for Human Health, Fribourg, Switzerland

\section{Authors' information}

Lavinia Alberi Auber, University of Fribourg and Swiss Integrative Centre of Human Health, Passage du Cardinal 13B, CH-1700 Fribourg, Switzerland. Email: lavinia.alberi@unifr.ch. Tel: 0041-26-3006512. Praveen Bathini, email: praveen.bathini@unifr.ch; Isabel Dupanloup, email: isabelle.dupanloup@sib.swiss, Elena Zenaro, email: elena.zenaro@univr.it, Eleonora Terrabuio ,email: eleonora.terrabuio@univr.it, Amrei Fischer, email: amrei.fischer@unifr.ch, Edona Ballabani, email: edona.ballabani@unifr.ch, Marie-Agnes Doucey, email: Marie-

Agnes.Doucey@ichnossciences.com.

\section{Abstract}

\section{Background}

Alzheimer's disease (AD) is the primary cause of cognitive deficit in elderly humans. Late-onset $A D(L O A D)$ is sporadic, multifactorial, non-Mendelian accounting at present for $95 \%$ of the cases in contrast to the genetic form. Risk factors for sporadic AD include Gene: Environment interactions. There is increasing evidence that lifestyle and environmental stress such as infection and chronic inflammation are underlying culprits of neurodegenerative dementia. Dementias that share or mimic pathological processes of $A D$ include cerebrovascular diseases, Lewy body disease, TDP-43 proteinopathy. To date, very few mouse models reproduce the pathophysiological progression of mixed-vascular-AD, while the majority of studies have employed transgenic animals reproducing the familial form.

\section{Methods}

We have re-engineered the Polyinosinic:polycytidylic acid (Polyl:C) sterile infection model in wildtype C57BI6 mice to obtain chronic low-grade systemic inflammation. We have conducted a cross-sectional analysis of aging Polyl:C and Saline control mice ( 3 months, 6 months, 9 months and 16 months), taking the hippocampus as a reference brain region, based on its vulnerability, and compared the brain aging phenotype to $A D$ progression in humans with mild $A D$, severe $A D$ and Controls (CTL), parallely in Vascular dementia (VaD) patient specimens.

\section{Results}

We found that Polyl:C mice display both peripheral and central inflammation with a peak at 6 months, associated with memory deficits. The hippocampus is characterized by a pronounced and progressive tauopathy. In Polyl:C brains, microglia undergo aging-dependent morphological 
rearrangements progressively adopting a phagocytic phenotype. Transcriptomic analysis reveals a profound change in gene expression over the course of aging, with a peak in differential expression at 9 months. We confirm that the proinflammatory marker Lcn2 is one of the genes with the strongest upregulation in Polyl:C mice upon aging. Validation in brains from patients with increasing severity of $\mathrm{AD}$ and $\mathrm{VaD}$ shows a reproducibility of some gene targets in vascular dementia specimens rather than AD ones, in which only GFAP is strongly increased at the severe stages.

\section{Conclusions}

The Polyl:C model of sterile infection demonstrates that peripheral chronic inflammation is sufficient to cause neuropathological processes resembling a mixed-VaD-AD phenotype, with progressive tau hyperphosphorylation, changes in microglia morphology, astrogliosis and gene reprogramming reflecting increased neuroinflammation, vascular remodeling and the loss of neuronal functionality seen to some extent in humans.

\section{Keywords}

Inflammation, Polyl:C, brain aging, microglia, sporadic Alzheimer's disease, Vascular dementia,

\section{Background}

Alzheimer's disease (AD) is the most common form of dementia, with vascular dementia (VaD) accounting for the second most common form. AD is a progressive neurodegenerative disorder characterized by amyloid- $\beta$ plaques and neurofibrillary tangles resulting in cognitive decline and neuronal loss. Most of the AD cases are familial and account for $5 \%$ of the cases whereas $95 \%$ cases are sporadic with a late-onset AD (Alonso, Grundke-lqbal, and lqbal 1996; Masters et al. 2015). While the familial $A D$ (FAD) has been linked mostly to the mutations in the amyloid precursor protein (APP) and presenilin-1 \&2 genes (PS1 and PS2), the etiology of late-onset or sporadic AD affecting people over 65 years of age is still not clearly understood. An increasing body of literature shows that mixed pathologies involving AD with cerebrovascular diseases, or Lewy body disease, or TDP-43 proteinopathy (10-78\% prevalence) (Rahimi and Kovacs 2014) with cerebrovascular and sporadic AD (over $20 \%$ of cases) representing the most common mixed pathology in advanced aging (Toledo et al. 2013; Lin et al. 2019; Santos et al. 2017). Scientific studies show the involvement of both genetic and environmental factors in sporadic AD. Some of these genes include ABCA7, APOE, BIN1, CD2AP, CD33, CLU, CR1, EPHA1, MS4A4A/MS4A4E/MS4A6E, PICALM, and SORL1 (Barber 2012), of which a large proportion are immunological factors. Modifiable risk factors for AD include inflammation, viral infections, Type 2 diabetes, vascular disorders, and hypertension (Edwards et al. 2019; Stozická, Zilka, and Novák 2007). Among these risk factors, inflammation is considered as a central mechanism in AD and infectious agents like bacteria and viruses might play a critical role in sustaining chronic inflammation and inducing neuroinflammation in particular in subjects carrying the aforementioned genetic variants. SORL1 and APOE have been also associated with Cerebral Amyloid Angiopathy (CAA) (Du et al. 2019) supporting vascular inflammation as comorbidity in AD. Notably, Cerebral Amyloid Angiopathy (CAA) is associated with higher CERAD scores, higher 
phase of $A \beta$ deposition, and higher Braak stages (Thal et al. 2002). Since the last decade, many epidemiological studies in humans have proposed the link between dysfunction of the immune system and AD pathogenesis (Morgan et al. 2019; Engelhart et al. 2004; Leung et al. 2013), yet much less attention has been given to mixed pathologies. Earlier studies indicate that peripheral inflammation can prompt central immune responses (Perry, Cunningham, and Holmes 2007) and an immune memory persisting in the brain (Saeed et al. 2014) causing neurodegeneration. Also, abnormal expression of these immune factors in response to stress or infection during pregnancy can lead to neurodevelopmental disorders and behavioral deficits in the offspring (Bilbo et al. 2018) and may underlie neurodegenerative processes later in life (Knuesel et al. 2014).

Here, we investigated pathophysiological response using a wild type mouse model exposed to viral-like immune activation, using Polyinosinic:polycytidylic acid (Polyl:C), in late pregnancy and the young offspring. The second immune challenge bolus was designed to achieve sustained chronic inflammation deviating from the original model (Krstic et al. 2012) that could not be replicated by other studies (Giovanoli et al. 2015). The new optimized Polyl:C mouse model reveals long term systemic \& central immune response resulting in significant proteinopathy associated with spatial memory deficit, microglia remodeling, transcriptomic changes leading to increased lipid metabolism and vascular factors activation, resembling a mixed vascular-AD pathophysiology.

\section{Materials and Methods}

\section{Animals}

Wildtype C57BL/6J mice were used for the study. Animal experimentation was approved by the animal experiment committee, University of Fribourg (Protocol no. 2016_32_FR registered 01/01/2017). The animals were fed ad libitum and housed in a room equipped with automatically controlled temperature $\left(21-25^{\circ} \mathrm{C}\right)$, humidity $(50 \%)$, and with a $12 \mathrm{hrs} \mathrm{light/} \mathrm{dark} \mathrm{cycle.}$

\section{Human tissue}

Frozen Human tissue samples from the entorhinal cortex including the hippocampal area were procured from the Medical Research Council Brain Bank for Dementia Research, Oxford, UK, and Stanford brain bank. We received frozen hippocampal brain tissue samples from 9 controls, 5 moderate, and 10 Severe sporadic AD patients (Suppl. Table 1). The use of human tissue has been approved by the Ethical Commission of the Brain Bank for Dementia UK (OBB443 registered 1/05/2017 and OB344 registered 1/02/2014), Stanford (Stanford IRB), and the Ethical Commission from the Canton of Fribourg and Vaud (N. 325/14). Vascular dementia hippocampal specimens and healthy age-matched controls were obtained from the Netherlands Brain Bank (NBB. 1251). All experiments conducted on human tissue comply with the WMA Declaration of Helsinki.

\section{Treatment}

To investigate the early postnatal treatment and microglial priming the systemic Poly I:C challenges were optimized from the already existing model with Polyl:C (Krstic et al. 2012). Female C57BL/6J mice 6-8 weeks old were housed together with the males for mating. Vaginal plugs were assessed and pregnant mice with gestation day (GD) 17 were injected intravenously (i.v.) with $5 \mathrm{mg} / \mathrm{kg}$ Polyl:C (Polyinosinic-polycytidylic acid; P9582, Sigma, USA). Aliquots of 5mg/ml 
were prepared by resuspending in the sterile $0.9 \%$ saline and were stored at $-20^{\circ} \mathrm{C}$ until further use. For control experiments sterile $0.9 \%$ saline was used. To mimic the chronic inflammatory conditions like in humans, prenatally challenged offspring were given a second immune challenge with an intraperitoneal (i.p.) Polyl:C injection at $20 \mathrm{mg} / \mathrm{kg}$ dose or sterile saline for the control experimental mice (Fig. 1A).

\section{Behavioural experiments}

Anxiety was tested at 3, 6, 9 and 16 months of age in PP and NN mice using the Open field test registering the time spent in the center, while elevated O-maze was used at 3 to 6 months only recording both the time spent in the open unprotected arm and time spent in the light. Working memory was tested using the Y-maze alternation task and expressed as \% of alternation and number of arm entries during a 5 minutes exploration-window according to previously published protocols (Crawley and Bailey 2008; Miedel et al. 2017). Mice during the behavior were videotaped and Videos were analyzed using Image $\mathrm{J}$-built in macros for automated video-tracking (Supplementary Material and Methods) (Brai and Alberi 2015).

\section{Tissue Processing}

Animals were first deeply anesthetized with pentobarbital sodium (100mg/kg, i.p), later toe pinch pain reflex was checked. After the abdominal opening, mice were transcardially perfused with $0.9 \%$ sterile saline, brains were harvested and cut into two hemispheres. One hemisphere was dissected to collect the hippocampus and further dissected in ice-cold saline solution to obtain the Corpus Ammonis (CA) fields, removing the dentate gyrus (Brai et al. 2015). The tissue samples were collected into eppendorfs and were flash-frozen in liquid nitrogen and stored at $80^{\circ} \mathrm{C}$ until further use. Another hemisphere was post-fixed in 4\% PFA for 1 day, followed by immersion in $30 \%$ sucrose at $4^{\circ} \mathrm{C}$ sucrose and then embedded in an OCT block for cryosectioning at $35 \mu \mathrm{m}$ thickness (Leica, Germany) and used for histological studies.

\section{Plasma collection}

The whole blood collected via cardiac puncture was transferred into the EDTA-coated eppendorfs. Cells were separated from the plasma by centrifugation for $15 \mathrm{~min}$ at $2000 \mathrm{xg}$ at $4^{\circ} \mathrm{C}$ before extracting the plasma. The plasma samples were then stored at $-80^{\circ} \mathrm{C}$ until further use.

\section{Isolation of leukocytes from mouse tissues and Flow cytometry}

Blood: Mice were anesthetized via isoflurane inhalation. Blood samples were collected through a retro-orbital not-surgical procedure by sodium heparinized capillaries. Dextran $1 \%$ and Heparin $10 \mathrm{U} / \mathrm{ml}$ were added to blood in a 1:1 ratio. After erythrocytes sedimentation (1 hour), the overlying supernatant plasma-dextran suspension of leukocytes was washed in 1X PBS. Red blood cell lysis was performed adding $3 \mathrm{ml}$ of $\mathrm{NaCl} 0.2 \%$ for 40 seconds and then $7 \mathrm{ml}$ of $\mathrm{NaCl} 1.2 \%$. Next, cells were washed and stained.

Brain: Mice were anesthetized and quickly perfused through the left cardiac ventricle by injection of cold PBS $1 \mathrm{X} \mathrm{Ca}_{2} \mathrm{Mg}_{2} 1 \mathrm{mM}$. After meninges removal, brains were collected. Mechanical digestion through gentleMACS ${ }^{\mathrm{TM}}$ Octo Dissociator (Miltenyi Biotec) and subsequent enzymatic digestion with $2 \mathrm{mg} / \mathrm{ml}$ of collagenase (C0130; Sigma-Aldrich, USA ) and $40 \mathrm{U} / \mathrm{ml}$ of DNase 
(EN0521; Invitrogen, Life Technologies, USA) at $37^{\circ} \mathrm{C}$ for 45 minutes in water bath were performed. Cells were isolated by passing the digested tissue through a $70-\mathrm{mm}$ cell strainer, resuspended in 30\% Percoll (GE Healthcare, USA), and loaded onto 70\% Percoll (GE Healthcare, USA). After centrifugation at $2500 \mathrm{rpm}$ for 20 minutes at $4^{\circ} \mathrm{C}$, cells were removed from interphase, washed, and stained.

Cells were treated with an antibody against Fc receptor [anti-mouse CD16/32, Clone: $2.4 \mathrm{G} 2$; Becton Dickinson (BD), USA] and labelled with BD Horizon Brilliant ${ }^{\mathrm{TM}}$ Stain Buffer (BD, USA) to improve staining quality and with $2,5 \mathrm{~mL}$ per sample of each of the following anti-mouse mAbs: Ly6G BB515 ( Clone: 1A8; BD, USA), CD45 BV786 (Clone, 104 RUO; BD, USA ), CD11b BV421 (Clone: R1-2; BD, USA), F4/80 (Clone: T45-2342 RUO; BD, USA), CD11c BV 605 (Clone: HL3; BD, USA), Siglec F PE (Clone: S17007L; BD, USA),B220 APC (Clone: RA3-6B2 RUO; BD, USA ). Cells were stained for 15 minutes at $4^{\circ} \mathrm{C}$ in the dark according to manufacturer's instructions. After washing, cells were incubated with 7AAD (BioLegend, USA) fluorescent intercalator for 5 minutes at $4^{\circ} \mathrm{C}$ in the dark. Samples were acquired through LSRFortessa X-20 (BD, USA). Data were analyzed through FlowJo ${ }^{\mathrm{TM}}$ Software. In particular, after doublets removal, 7AAD- alive cells were selected. A specific gating strategy was used to identify subpopulations of leukocytes in blood and brain samples. The following $\mathrm{CD}_{4} 5^{+}$cell populations were detected and analyzed: Ly6G+ CD11 b+ B220- Neutrophils, Siglec F+ CD11b- CD11c- Eosinophils, F4/80+ CD11 b ${ }^{+}$SiglecFMonocytes. Using the DownSample Plugin developed by FlowJo ${ }^{\mathrm{TM}}$ (https://docs.flowjo.com/seqgeq/dimensionality-reduction/downsample/), the number of CD45+ events in data matrix was reduced to a maximum of 30.000 .

\section{Bead-based Immunoassay}

To study the Polyl:C effect on plasma inflammatory profile, we measured chemokines IL-6, IL-10, MCP-1, and TNF- $\alpha$ using the premixed inflammation panel (PN: C282251A, Aimplex Biosciences, USA) according to the manufacturer's instructions. Briefly, $45 \mu \mathrm{l}$ of the capture bead working solution, $30 \mu \mathrm{l}$ of assay buffer, and $15 \mu \mathrm{l}$ of the sample were incubated on the shaker (700 rpm) for 60 minutes at room temperature. After 3 washes with $100 \mu$ l washing buffer, $25 \mu l$ of biotinylated antibody working solution was added and incubated on the shaker for 30 min at room temperature with protection from light. After 3 washes, $25 \mu \mathrm{L}$ of streptavidin-phycoerythrin working solution was added, shaken for 20 minutes in the dark. Finally, the beads were resuspended on $150 \mu \mathrm{l}$ of $1 \mathrm{x}$ reading buffer and read on Flow Cytometry Analyzer (LSR-Ila; BD, USA) acquiring about 50 events per chemokine. The FCS files were analyzed on Flowing software (http://flowingsoftware.btk.fi/) and the inflammatory levels were determined based on 5-parameter logistic curve fitting.

\section{Immunoassay of Amyloid- $\boldsymbol{\beta}_{1-42}$}

Frozen cortical tissue samples were homogenized with lysis buffer containing $20 \mathrm{mM}$ Tris- $\mathrm{HCl}$ (pH 7.5), $150 \mathrm{mM} \mathrm{NaCl}, 1 \mathrm{mM}$ disodium EDTA, $1 \mathrm{mM}$ EGTA, 1\% Triton, $2.5 \mathrm{mM}$ sodium pyrophosphate, $1 \mathrm{mM} \beta$-glycerophosphate (G9422; Sigma, Germany), phosphatases inhibitor such as Sodium Orthovanadate (1 mM, 450243; Sigma, Germany) and proteases inhibitor cocktail 1:100 (3749.1, Roth, Germany) and the lysates were stored at $-80^{\circ} \mathrm{C}$ until further analysis. The sample and reagents preparation has been carried out according to the Milliplex Map kit Human 
Amyloid and Tau Magnetic Bead panel (Cat.\# HNABTMAG-68K) manufacturer's protocol. Briefly, cortical lysates were diluted 1:2 with assay buffer. Standards, controls and samples were added to the appropriate wells of the 96 well filter plate. Biotinylated detection antibodies and magnetic mixed antibody-immobilized beads were added, sealed with aluminum foil, and incubated overnight in the dark at room temperature on a shaker (700rpm). The next day following washes, Streptavidin-phycoerythrin was added and incubated in the dark for 30 minutes at room temperature on a shaker (700rpm). Finally, the beads were suspended in $100 \mu \mathrm{l}$ Sheath fluid and transferred to the 96 well flat bottom plate to be read on the FlexMap 3D system equipped with xPONENT software (Merck, Germany). The median fluorescence intensity (MFI) was analyzed using a 5-parameter logistic curve-fitting method and analyte concentrations were calculated.

\section{Nucleic acid and protein extraction}

Fresh frozen hippocampal tissue specimens from mice and humans are stored at $-80^{\circ} \mathrm{C}$ until utilized for RNA extraction using peqGOLD TriFast ${ }^{\mathrm{TM}}$ (peqGOLD, Germany) according to the manufacturer's instructions to obtain RNA and proteins. The RNA pellet was extracted from the aqueous phase, air-dried, resuspended in $25 \mu \mathrm{l}$ of nuclease-free water (Promega). The concentration of the RNA samples was measured using the Qubit 3.0 Fluorometer (High sensitivity, Invitrogen) and stored at $-80^{\circ} \mathrm{C}$ until further use. Proteins were extracted from the organic phase, cleaned with Ethanol, and the dried pellet resuspended with a $150 \mu \mathrm{l}$ buffer containing $8 \mathrm{M}$ Urea in 4\% (w/v) CHAPS and protease inhibitor (1:100 Carl Roth, Germany). The protein concentrations were determined using the Bradford assay method (Roth, Germany) and later the samples were stored until use at $-80^{\circ} \mathrm{C}$.

\section{RNA library preparation and sequencing}

Synthesis and amplification of cDNA were performed as per the Illumina TruSeq Stranded mRNA protocol. RNA extracted from the hippocampal specimens from all the age groups $(3 \mathrm{~m}, 6 \mathrm{~m}, 9 \mathrm{~m}$, $16 \mathrm{~m})$ was used for this transcriptomics study. RNA integrity was determined with the Fragment Analyzer 5200 (Agilent). Samples with RNA integrity number (RIN) > 8 were used for the experiment. An input material of $1 \mu \mathrm{g}$ of total RNA from each sample was used for library preparation with Illumina TruSeq Stranded mRNA kit (Cat: 20020595). Illumina TrueSeq Combinatorial dual (CD) indexes were used during the ligation, DNA fragments enriched using the PCR to amplify the amount of DNA in the library. The quality of the libraries is determined using the Standard High sensitivity NGS Fragment analysis kit (DNF-474, 1-6000 base pair) on the Agilent Fragment analyzer (Agilent, USA), yielding approximately 260 bp size fragments. The cDNA libraries were pooled in equivalent amounts. The libraries were denatured and diluted using standard library quantification and quality control procedures recommended as per the NextSeqprotocol. For a sequencing control, PhiX library was prepared and combined with the pooled prepared libraries. A final concentration of $1.5 \mathrm{pM}$ library was sequenced on Illumina NextSeq 500 system (High output 150 cycles) to generate 20 million of $2 \times 75$ bp pair-end reads per library. 


\section{Bioinformatics Analysis}

In brief, hippocampal paired-end libraries from all the age groups $(3 \mathrm{~m}, 6 \mathrm{~m}, 9 \mathrm{~m}, 16 \mathrm{~m})$ in the saline treated and Polyl:C treated mice ( $n=3$ each) were sequenced for the study. To generate the differentially expressed transcripts from the RNA sequencing data the following bioinformatics analysis pipeline was performed. 1) Remove adapter sequences and remove low quality (flanking $\mathrm{N}$ ) bases from each read using cutadapt version 2.3; 2) Alignment of the reads to the reference genome using RNA-seq aligner STAR version 2.6; 3) Get basic alignment stats with RSeQC version 2.6 .4 ; 4) Get read distribution with RSeQC version 2.6.4; 5) Get gene body coverage with RSeQC version 2.6.4; 6) Get read counts for genes with htseq-count release_0.11.1; 7) perform differential expression analysis with DESEQ2.

Further functional enrichments were performed using the ClueGO (Bindea et al. 2009) which integrates Gene ontology (GO), KEGG ( Kyoto Encyclopedia of Genes and Genomes), Wikipathway, Reactome pathway analysis creating a functional pathway network.

\section{Reverse transcription-polymerase chain reaction (RT-PCR)}

$1 \mu \mathrm{g}$ of total RNA from the human and mouse brain specimen was reverse-transcribed using the M-MLV reverse transcriptase (Promega) for efficient synthesis of the first-strand cDNA. Gene expression analysis was done by RT-PCR (GoTaq qPCR Master Mix, Promega, USA) using gene specific primers (Suppl. Table 2) on Mic qPCR Cycler (BioMolecular Systems, USA). Expression levels of genes of interest were determined using the $\triangle \triangle C T$ method; the levels of the mRNAs of interest were normalized against the levels of the housekeeping gene, $\beta$-actin.

\section{Antibodies \& reagents}

Following antibodies were used for western blot immunolabeling, immunohistochemistry experiments in this study:

Rabbit anti pTau 231 (1:500, ab151559, Abcam, UK), Rabbit anti pTau 205 (1:500, ab181206, Abcam, UK), goat anti-tau (1.25 $\mu \mathrm{g} / \mathrm{mL}$; AF3494, R\&D systems, USA), mouse anti- $\beta$ actin, 1:2000 (sc-81178; Santa Cruz Biotechnology, USA), Rabbit anti GFAP (1:500, ab68428, Abcam, UK), Goat anti GFAP (1:500, SAB2500462, Sigma), Goat anti lba1 (1:500, ab5076, Abcam, UK), Mouse anti-amyloid beta 6E10 clone (1:500, cat:803014; Biolegend, USA), rabbit antisynaptophysin (1:500; ab14692, Abcam, UK), Rabbit anti-amyloid beta 42 (Aß-42; 1:500; D54D2 XP, Cell Signaling, USA), Rabbit anti-Lipocalin-2/LCN2 (1:500, 50060-RP02; Sino Biological, China), mouse anti-NF200 (1:500, cat:1178709; Boehringer Mannheim Biochemica, Germany), Nile Red (2 $\mu \mathrm{g} / \mathrm{mL}$, N3013, Sigma, USA), Thioflavin-S (100 mM; CAS 1326-12-1, Santa Cruz Biotechnology, USA)

The secondary antibodies used in the study for immunolabeling were: directly conjugated to Cy2, Cy3, or Cy5 in donkey raised secondary antibodies (all 1:1000; Jackson Immuno Europe, UK). After the completion of immunofluorescence protocols, the sections were stained with DAPI to visualize nuclear morphology, mounted on Super frost slides (Thermofisher, USA), and coverslipped with a custom made Polyvinyl alcohol (PVA) and 1,4-diazabicyclo[2.2.2]octane (DABCO)-based mounting media.

\section{Western blot}


Hippocampal lysates supplemented were denatured at $95^{\circ} \mathrm{C}$ using the 2-Mercaptoethanol based loading buffer. Proteins were separated using the SDS-polyacrylamide gel electrophoresis and western blot procedure. Custom made $8-10 \%$ gels were used to run the samples for 1.5 hours at 110 voltage. The proteins were then transferred to a precut $0.2 \mu \mathrm{m}$ nitrocellulose membrane (Cat: 1620146; Biorad, USA) using a wet transfer method (Biorad). Later the membranes were incubated in a blocking solution containing $0.5 \%$ bovine serum albumin (Art. No. 8076.4, Roth, Germany), 1xTBS, and 0.1\%Tween-20 for 30 minutes at room temperature on a rotating shaker. The membranes were then incubated with primary antibodies overnight at $4^{\circ} \mathrm{C}$ with gentle agitation. On the second day, the membranes were rinsed with TBS-Tween solution $3 \times 5$ minutes, followed by a 1-hour incubation with the fluorescently conjugated secondary antibodies, rinsed again and air-dried covered by aluminum foil. The proteins were detected using the Omega Lum (Labgene, $\mathrm{CH}$ ). The optical density of the protein bands was determined using Image $\mathrm{J}$ software and normalized to the Beta-actin control. For an accurate total protein quantification REVERT stain (LI-COR Biosciences-GmbH, Germany) was also employed.

\section{Fluorescent immunohistochemistry}

The mice sagittal tissue sections from the anti-freezing medium were taken and then mounted onto the superfrost glass slides, air-dried for 1 hour, and washed twice in Distilled water 5 minutes each. To access epitopes slides were incubated at $65^{\circ} \mathrm{C}$ (water bath) for 20 minutes in $10 \mathrm{mM}$ Sodium citrate $(\mathrm{pH} 6)$ containing $0.05 \%$ Tween (Preheat buffer). Thereafter, sections were washed 3 times 5 minutes each in $1 \times$ Trizma-based salt solution (TBS), at room temperature, and once in a TBST buffer ( $1 x$ TBS containing $0.1 \%$ Triton solution). Sections were blocked in the blocking solution (TBST buffer containing 10\% fetal bovine serum) for 1 hour at room temperature in a humid chamber. Further, the sections were incubated with the primary antibody in the TBST buffer containing $1 \%$ fetal bovine serum at $4^{\circ} \mathrm{C}$ in the refrigerator overnight. The next day, sections were washed 3 times for 5 minutes each in the TBS buffer before being incubated with the fluorescently labeled secondary antibodies for 3 hours at room temperature. Following the labeling, the sections were washed 3 times in 1x TBS buffer, 5 minutes each. In one staining (pTau and Thioflavin S), sections were incubated with Thioflavin S, followed by $2 \times 5$ minutes washes with distilled water. After the last wash, sections are incubated with DAPI (4',6-diamidino2-phenylindole, Cat. no. 10236276001 Roche, Switzerland) for 10 minutes at room temperature. Thereafter, the sections were washed 2 times, 5 minutes each with TBS, and mounted with custom-made aqueous mounting media containing 1,4-Diazabicyclo[2.2.2]octane (803456 EMD Millipore, USA). For the lipid droplet Nile red staining, sagittal brain sections were mounted on to the glass slide and air-dried, followed by a rinse with deionized water for 1-2 minutes. Nile $\mathrm{red} / \mathrm{glycerol}$ staining solution $(2 \mu \mathrm{g} / \mathrm{ml})$ was added onto the tissue, incubated for 5 minutes, and coverslipped before being examined using a confocal fluorescence microscope (Zeiss LSM 800, Germany).

\section{Image quantification}

To quantify the glial response to an increase in systemic and central inflammatory profile, the morphology of these brain innate immune cells has been analyzed. Iba-1 positive microglia images (10 ROl/section) were taken at 40x oil immersion objective using a Confocal microscope (Zeiss LSM 800, Germany) with $1 \mu \mathrm{m} \mathrm{Z} \mathrm{intervals} \mathrm{resulting} \mathrm{in} \mathrm{stacks} \mathrm{of} \mathrm{20-30} \mathrm{slices} \mathrm{at} 512 \times 512$ pixel resolution. The soma area, perimeter, circularity, skeleton analysis was performed as 
instructed (Young and Morrison 2018; Davis et al. 2017) and using custom-made macros (Suppl. Materials and Methods). For the Fractal analysis on aged mice microglia 60x Oil immersion objective was used to generate in-depth microglia morphological data. Mean fluorescence intensity (pTauT231) of the CA1 field was measured in the same age group using the ImageJ ROI manager. Amyloid and Lcn2 staining were analyzed for \% area coverage using the custom made ImageJ macros (Suppl. Materials and Methods). Nile red positive lipid droplets were analyzed using the Analyze particle function in ImageJ. For the histological data, ImageJ macros were created and used for the analysis for an unbiased morphological quantification (Suppl. Materials and Methods). For the western blot, protein band images were analyzed using the ImageJ Gel quantification tool.

\section{Statistical analysis}

Homogeneity of variance for each variable within a group/ age was verified by Levene's Test and comparative analysis performed either using the parametric test for normally distributed data (Student's t-test) or non-parametric test for non-normally distributed data (Mann Whitney test). Statistical significance was assessed using unpaired, 2-tailed Student's t-test. Results (mean \pm SEM) were obtained using Excel Real Statistics plugin. $P$ values less than 0.05 were considered significant. RNA sequencing data were analyzed using the ` $\mathrm{`}$ 'DESEQ2 package and statistically significant transcripts were chosen if $p<0.05$ and $p$ adjusted for multiple testing $<0.1$. For the flow cytometry data, statistical analysis was performed through unpaired Welch's t-test using GraphPad Prism Version 8.1.2. Optical densities of immunoblots were compared between groups of the same age using Student's t-test. Morphology measurements of microglia were analyzed using the Kolmogorov-Smirnov test and in aggregate using Student's t-test. Correlation analysis was conducted using $\mathrm{R}$ and significance tested using Student's t-test.

\section{Results}

The goal of the study is to demonstrate that low-grade chronic inflammation, through prenatal and postnatal sterile infection with Polyl:C is sufficient to cause an AD-like pathology.

\section{Chronic inflammation after double Polyl:C challenge}

To obtain systemic low-grade chronic inflammation we have performed double sterile infection with the TLR3 (Toll-like receptor 3) stimulant, Polyl:C, in the mother at gestational day 17 and in the offspring at 2.5 months (PP). This protocol deviates from the previous studies using either prenatal injection alone or the combination of prenatal and senior injections (Krstic et al. 2012), which could not be replicated by others (Giovanoli et al. 2015) and aims at investigating progressive pathophysiological changes in response to inflammation already in midlife. Animals were analyzed cross-sectionally at 3,6, 9, and 16 months, taking saline-injected controls as reference (NN) (Fig. 1A). From 3 to 6 months of age levels of four circulating inflammatory cytokines (MCP-1, IL-6, TNF- $\alpha$, and IL-10) are progressively higher in PP as compared to NN (Table 1). At 9 months, all 5 cytokines are increased already in the NN, as compared to the previous stages and only IL-6 is 6 fold elevated in PP mice $(p<0.05)$ (Table 1). At 16 months, NN and PP mice show no difference in humoral immune factors (Table 1). Considering the upregulation of a large population of circulating chemokines in the blood of PP mice, we examined the neutrophils, monocytes, and total polymorphonuclear cells (PMNs) from the freshly isolated 
whole blood and brain samples to study any infiltration of these leukocytes into the brain. Samples were analyzed using the flow cytometry for the expression of neutrophils and monocytes using antibodies stained for Ly6G, Siglec, F4/80, CD11b. Although no changes are observed in the 3 months of age between groups, at 6 months PP mice exhibit elevated levels of neutrophils and PMNs in the systemic circulation but no significant differences are seen in the brain of PP mice as compared to NN animals (Suppl. Fig. 1A and B). On the other hand, the monocyte population differs neither in the blood nor in the brain (Suppl. Fig. 1C). While there is no trace of infiltrating immune cells in the brain at 3 or 6 months, analysis by RT-PCR of IL- 6 transcripts shows a significant increase in PP mice at 6 months, which then stabilizes at 9 months and decreases at 16 months compared to $N N$ controls $(p<0.01)$ (Fig. 1B). We further analyzed how the inflammatory transcripts correlate with cell-type-specific transcripts for neurons (GRIN1, Glutamate lonotropic Receptor NMDA Type Subunit 1), microglia (Iba1; lonized calcium-binding adaptor molecule 1) and astroglia (GFAP; Glial Acidic Fibrillary Protein) in PP and NN animals. We observe that GRIN1 expression is stable except for a trending increase at 9 months (Fig. 1C), whereas Iba1 shows dynamic changes with a progressive increase from 6 to 9 months and drop at 16 months $(p<0.01)$ (Fig. 1D) similarly to the proinflammatory cytokines $\left(1 \mathrm{IL}-6, \mathrm{r}_{16 \mathrm{mo}}=0.77\right.$ and $\left.\mathrm{IL}-1 \beta, \mathrm{r}_{16 \mathrm{mo}}=0.68\right)$. At 6 and 9 months also, GFAP expression increases but decays to normal levels at 16 months (Fig. 1E). The temporal evolution of GFAP overlaps with that of IL-1 $\beta,(r=0.62$, $\mathrm{p}<0.05$; Fig. 1F). Our results show that the combination of prenatal and an early postnatal Polyl:C immune activation causes a prolonged and sustained systemic inflammatory response with the recruitment of circulating leukocytes of the innate immunity, and increased neuroinflammation and gliosis at mid-age.

\section{Progressive tauopathy in PP mice}

Inflammatory mechanisms are central to AD pathology involving both the interaction between inflammatory stimuli, proinflammatory cytokines/mediators, and AD pathological hallmarks such as aggregates of amyloid-beta $(A \beta)$ and hyperphosphorylated tau protein ( $p$-tau) (Kinney et al. 2018), underlying neural network dysfunction and brain atrophy. We determined the impact of systemic immune challenge on the pre-tangle pathology by analyzing tau hyperphosphorylation at positions 231 and 205 using western blot (Fig. 2A) and immunohistochemistry (Fig. 2F and Suppl. Fig. 2A) on hippocampal tissue from 3 to 16 months of age. From the immunoblot, the content of hippocampal p-tau (pTau T231) relative to total tau (ptau/tau) increases dramatically in PP mice at 6 months, remains elevated at 9 months and then is indistinguishable from NN mice at 16 months (Fig. 2B and 2C). The progressive tauopathy is confirmed by immunolabeling of pTau fibers (pTau T205) in the hippocampal CA1 region and CA1 stratum lacunosum (Suppl. Fig. 2A), with a significant increase in pTau pixels in the CA1 region at 16 months (Suppl. Fig. 2B). Tau hyperphosphorylation and the formation of $\beta$-sheet fibrils, labeled by Thioflavin-S, can be observed in the CA3 field at 9 months in PP mice, in contrast to NN (Fig. 2F). To assess whether the progressive tauopathy in PP is associated with synaptic abnormalities and neuroinflammatory responses, we quantified the levels of synaptophysin and GFAP overtime (Fig. 2D). At 16 months in PP mice, we observe a drop in synaptophysin expression accompanied by an increase in GFAP (Fig. 2E). The increase in GFAP immunoreactivity is confirmed by immunohistochemistry of the CA3 region showing the presence of amyloid- $\beta$ 1-42 labeled by an antibody recognizing the amino acid region 1-16 (A $\beta 1-16)$ and $p$-tau aggregates (Fig. $2 \mathrm{G})$. Further analysis of amyloid- $\beta$ fibrils in 
the 16 months old mice indicates that $A \beta 1-42$ is also present in NN old mice with internalization in GFAP positive glial cells, which is more pronounced in PP mice (Fig. 3A, white stars). In addition in 16 months PP mice, $A \beta 1-42$ positive aggregates are visible in vessels ensheathed by astroglial endfeet reflecting a cerebral amyloid angiopathy (CAA) (Fig. 3A, white arrows). Increased colocalization between insoluble $A \beta 1-42$ and $p$-tau in 16 months old PP as compared to NN both in the hippocampus (Fig. 3A) and the entorhinal cortex (Fig. 3B). In the latter region, A $\beta 1-42 / p$-tau positive aggregates display a stellate morphology resembling core-plaques (Fig. 3C, insert). The number of plaques rises 4 folds in PP as compared to NN (Fig. 3D), with a 70\% larger amyloid- $\beta$ burden in the area examined (Fig. 3E). Quantification of soluble A $\beta 1-42$ from the entorhinal cortex shows a peak in $A \beta_{1-42}$ release at 6 months in PP mice which later subdues (Fig. 3F), possibly as a result of insoluble aggregate formation in the brain.

\section{Polyl:C-induced memory impairment}

Based on the proteinopathy in the hippocampal and entorhinal cortex region following double Polyl:C injection, we assessed whether spatial working memory is affected. Previous work has shown that spontaneous alternation, a form of working memory, is entirely dependent on hippocampal synaptic function (Pioli et al. 2014; McHugh et al. 2008). PP and NN animals were subjected to the $Y$-maze spontaneous alternation task at 3, 6, 9, and 16 months. The number of arm entries and sequence were recorded, and the percent alternation was calculated (Fig. 3G). Although the activity of PP treated animals increases in terms of alterations at 3 months, the percentage of alternation did not change at this age as compared to $N N(t 17=0.76, P=0.46 n$ $=10$ for Control and $n=9$ for Polyl:C). On the other hand, deficits in the spontaneous alternation are observed in the PP group both at 6 months ( $t 17=3.21, p=0.005, n=10$ for NN and $n=9$ for PP), 9 months (t15 = 2.57, $\mathrm{p}=0.013, \mathrm{n}=9$ for $\mathrm{NN}$ and $\mathrm{n}=8$ for $\mathrm{PP}$ ) and 16 months (t13 $=2.57, \mathrm{p}$ $=0.02 n=8$ for $N N$ and $n=7$ for PP). The number of arm entries in the $Y$ maze task is not significantly different across all the age groups (Suppl. Table 4) indicating normal locomotory behaviour representing a non-confounding factor for the percentage of an alternation. However, from 9 months of age both PP and NN display about half of the number of entries as compared to younger animals. Since early and late gestational Polyl:C treatment has been linked to stress and anxiety-like phenotypes relevant to schizophrenia (Hui et al. 2018; Silveira et al. 2017) we tested these mice for Light/dark, Elevated O-maze at 3 and 6 months and Open field tasks at all ages. We found no difference in the anxiety-like behaviour (Suppl. Table 5) disambiguating the spatial memory impairments observed. Together the data suggest that prenatal and early postnatal viral-like immune activation through Polyl:C treatment causes proteinopathy of the limbic regions with sustained working memory impairment.

\section{Activation and phenotypic change in microglia of PP animals}

Earlier studies have shown that systemic infections and the subsequent peripheral immune activation have a strong effect on brain function, glial response representing a risk for dementia (Cunningham and Hennessy 2015; Cunningham 2013). Microglia cells are the primary phagocytic innate immune cells of the brain that get stimulated upon immune activation. They normally exist in a resting state displaying a ramified morphology, while in intermediate states display a bipolar or rod-like phenotype (Davis et al. 2017) and in an activated state an amoeboid, irregular shape (Ling and Wong 1993). Quantification of lba-1 positive cells per $\mathrm{mm}^{3}$ in the immunostained 
hippocampus (Fig 4A), indicates no difference between PP and NN at the different stages (Suppl. Table 6). Next, we assessed whether the PP brains show altered microglial cell morphologies reflecting an activated inflammatory status. We quantified morphological parameters like soma size, perimeter, circularity, skeleton analysis of lba-1 positive microglia (Fig. 4), and finally we conducted the fractal analysis in NN \& PP mice across staging. From 6 months of age, lba-1 positive microglia show an increased ramified morphology as detected by immunofluorescence microscopy (Fig. 4A). Quantitative soma analysis shows a slight increase in the soma size, typical of activated microglia in PP mice at 3 months $(22 \%, p<0.005)$, which last up to 9 months $(58 \%$, $\mathrm{p}<0.005$ (Fig. 4B). The cell soma perimeter measurement shows dynamic changes at 3 months $(11 \%$ increase, $p<0.01)$, and a significant increase at 9 months $(57 \%, p<0.005)$, while no changes are detected at 6 and 16 months (Fig. 4B). Circularity, which indicates the roundness index, shows a deviation from circularity in the intermediate ages at 6 months $(10 \%$ decrease, $p<0.005), 9$ months $(21 \%$ decrease, $p<0.005)$ and 16 months $(10 \%$ increase, $p<0.05)$, indicating signs of progressive microglial activation with aging (Fig. 4B). These dynamic changes were further analyzed using the distribution curve analysis which indicates an overall shift in the number of cells with an increase in microglial cells soma size and perimeter (Suppl. Fig. 3A-3B) with irregularly shaped cell body during the adult and mid-aged intermediate stages (6 and 9 months) while at later stages (16 months), microglia become more circular or rounded suggesting morphological fluctuations across staging and signs of microglial activation (Suppl. Fig. 3C). To further characterize the phenotypic changes in microglia, reflecting their activation state, we performed the skeletal analysis of Iba-1 positive microglia, at 3, 6,9, and 16 months in PP and NN mice. Skeletonized lba-1 renderings reveal a significant increase in the number of endpoints/cell, maximum branch length/cell, and branch length/cell (Fig. 4C) in PP mice with age groups 3 months to 9 months indicating hyper ramified microglia. However, at 16 months, microglia in PP brains show a significant drop in the number of endpoints/cells and an overall decrease in the branch and maximum branch length (Fig. 4C). Therefore, prenatal and early postnatal systemic Polyl:C immune activation induces hyper ramified microglia in the offspring which later undergoes a transition from hyperactivated to more bushy or amoeboid with the reduction in microglial endpoints.

Having detected a microglia morphological transition in aged PP mice, confocal imaged microglia cells were further investigated using fractal analysis (FracLac plugin of ImageJ) which renders the shape of microglia cells and quantifies the parameters of cell area, cell perimeter, Span ratio, circularity, and fractal dimension, the latter reflecting pattern complexity. The fractal analysis revealed significant changes in hippocampal microglia parameters in the aged 16 months PP mice versus NN (Fig. 4D). Normal resting microglia are complex with a higher fractal dimension. In the PP mice total microglia cell surface area, the perimeter is reduced indicating a more compact shape (Fig. 4E-4F). Fractal dimension as a measure for complexity and circularity as a measure for the roundness is also reduced after immune activation (Fig. 4H-4I). On the other hand, the span ratio is increased reflecting microglia elongation (ratio of cell length and width) (Fig. 4G). Span ratio and circularity are inversely proportional indicating an activated microglia state. Altogether, systemic inflammation through pre- and post-natal Polyl:C induces profound changes in microglia morphology indicating a shift from resting to an activated state in aged PP animals. 


\section{Dynamic remodeling of the hippocampal transcriptome}

To understand the mechanisms underlying the spatial memory deficits and the pathophysiological processes associated with the proteinopathy, and neuroinflammation we performed a crosssectional hippocampal bulk mRNA sequencing on 3, 6, 9, and 16 months PP and NN animals. The analysis reveals many differentially expressed genes (DEGs) in the hippocampus (log2 fold change cut-off:05, padjusted $<0.05$ ) between treatments. The gene expression profiles as visualized in the volcano plot (Fig. 5A-5D), show that in aged animals differential expression is highest with a peak at 9 months, coinciding with the neuroinflammatory switch in microglia cells. A GO analysis contextualized to the synapse (SYNGO) (Koopmans et al. 2019) indicates a dynamic shift in DEGs from the presynaptic compartment at 3 months (Supp. Fig 4A) to the postsynaptic terminal at 9 and 16 months (Supp. Figure 4C and 4D), with a non-synaptic stage at 6 months (Supp. Fig 4B). At 3 months, out of 35 DEGs between PP and NN, 21 are downregulated, and 14 are upregulated (Fig. 5A). Gene ontology enrichment analysis (GEA) of the biological processes using a $5 \%$ false discovery rate indicates general repression in genes associated with synaptic transmission, calcium signaling, extracellular matrix organization, and secretion (Suppl. Table 7). Pathway analysis based on a composite KEGG, Reactome, WikiPathways dataset shows high interconnectivity between cellular cascades associated with extracellular matrix organization, chemical synaptic transmission, and calcium signaling (Suppl. Fig 5A). In the 6 months, out 32 DEGs between PP and NN (Fig. 5B), 22 are downregulated, and 10 upregulated. A GEA indicates ongoing processes of morphogenesis and gliogenesis (Suppl. Table 7), with highly overlapping pathways (Suppl. Fig. 5B). At 9 months (Fig. 5C) out of 196 DEGs, 90 are downregulated, and 106 are upregulated. The GEA shows enrichment in several processes associated with vascular remodeling, neurogenesis, morphogenesis, cytokine response, and cell death (Suppl. Table 7). Pathway analysis indicates partially overlapping cellular cascades shared among focal adhesion, BMP signaling, MAPK signaling, and neuronal injury (Suppl. Fig. 5D) confirming the concurrent processes of morphogenesis and cell death at this stage. In the 16 months PP mice (Fig. 5D) from the 99 DEGs, 50 genes are downregulated and 49 genes are upregulated. GEA of the DEGs indicates enrichment in the response to metal ions, the reactive oxygen response, morphogenesis, the regulation of cellular proliferation, and the response to hormones (Suppl. Table 7). Pathways analysis shows less interconnected cascades implicated in potassium ion transmembrane transport, monocytes proliferation, lamellipodium organization, and glucose metabolism (Suppl. Fig. 5D).

To better understand the dynamic changes in the sterile infection model undergoing with age, we compared the significantly differentiated genes between PP and NN at the cross-sectional time points. As shown in the Venn diagram (Fig. 5E), the number of common genes that are uniquely and commonly affected in the hippocampus of 3, 6, 9, 16 months PP mice indicates a large number of overlapping gene sets between 9 and 16 months, while fewer genes are shared with earlier stages. In particular, 6 shared genes can be subdivided into 2 categories reflecting a progressive cell-communication dysfunction (I) and a proinflammatory drive (II) in aging PP mice. (I) Genes downstream of MAPK-signalling (c-Jun) (E. K. Kim and Choi 2010), NFKB-signaling (Egr2; Early growth response protein 2) (Williams et al. 1995), responsible for neuronal excitability (Kcnj2; Potassium Inwardly Rectifying Channel Subfamily J Member 2) (Binda et al. 2018), with reported association with synaptic dysfunction, are downregulated in PP mice starting from 6 
months (Fig. 5F). (II) On the other hand, genes related to neuroinflammation such as the lipiddroplet dependent gene (Plin4; Perilipin 4) (Han et al. 2018), pro-inflammatory genes (H2-Aa; Immunohistocompatibility-complex) (Van Hove et al. 2019) and acute-phase proteins regulating to the inflammatory response (Lcn2; Lipocalin-2) (Dekens et al. 2020) are upregulated in aging PP mice, starting from 9 months (Fig. 5G). Overall, the gene remodeling across the aging continuum replicates processes typical of $A D$ as neuronal network breakdown, altered immune response, chronic neuroinflammation and vascular dysfunction.

\section{Substantial changes in brain metabolism and inflammation}

We further validated the RNA-seq analysis via RT-PCR, on some of the significantly DEG with a reported association to AD as well as genes belonging to the neuronal (I) (Fig 5H) and metabolic (II) (Fig 5I) gene groups. At 3 months, Cacna1g (Calcium Voltage-Gated Channel Subunit Alpha1 $\mathrm{G}$ ), which was previously reported to decay with aging and regulate amyloid- $\beta$ production (Rice et al. 2014), is unchanged (0.9) in contrast to the observed reduction via RNA-seq (Log2FC=$0.55)$, suggesting that aggregate changes rather than single-gene changes may contribute to the modeled conductivity dysfunction (Suppl. Tab. 6). As expected at this stage, Plin4 and Egr2 are unchanged, matching the RNAseq data (Table 2). At 6 months, we analyzed one of the genes with the strongest downregulation at the RNA-seq, with reported association with cognitive impairment in chronic cerebral hypoperfusion (Xie et al. 2018). The Glpr2 (Glucagon-Like Peptide 2 Receptor) decrease (80\%), is confirmed (Table 2; $\mathrm{p}<0.05)$. Also, Ide (Insulin-degrading enzyme), which has been implicated in the clearance of insulin and amyloid- $\beta$ (Qiu and Folstein 2006), show a comparable increase, between RNA-seq and RT-PCR analysis, but did not reach significance in the RT-PCR result (Table 2; $p=0.16$ ). Interestingly, Kcnj2 show a significant 2 fold increase at 6 months (Table 2; $\mathrm{p}<0.05$ ), suggesting a modulatory $\mathrm{K}+$ currents effect. Plin4 remain unchanged between PP and NN at 6 months in both analyses (Table 2). At 9 months, the reduction in c-fos, c-Jun, Notch1, Kcnj2, Egr2, and the increase in Lcn2 can be confirmed at the RT-PCR, while Plin4 show a non-significant increase using RT-PCR (Table 2; $p=0.24$ ). At 16 months, Lcn2, Plin4 trends are reproduced according to the RT-PCR, while Kcnj2 shows a comparable but not significant decrease (Table 2; $p=0.36$ ). The data indicates that around $3 / 4$ of the RNA-seq outputs could be replicated via RT-PCR validation, confirming the robustness of the bulk RNAseq discovery method and emphasizing that aggregate dataset can explain ongoing cellular and molecular processes in such models. Validated DEG profiles of Kcnj2, Egr2, and Plin4 and Lcn2 are matched with the expression of genes specific for neurons (Grin1), microglia (Iba1) and astroglia (GFAP) at 9 and 16 months to investigate associations of the selected genes in specific cell types (Fig. 5J). Grin1, Iba1, and GFAP are positively associated in their peaking trend at these time points (Fig. 1C-E) supporting the neuro-glia interplay. On the other hand, Egr2 which decreases at 9 months and increases at 16 months is inversely correlated to Grin1, Iba1, and GFAP suggesting a potential ubiquitous regulation of this transcription factor on the cell-typespecific changes. Finally, Plin4 and Lcn2 are positively associated with their increasing trend from 9 to 16 months, confirming the RNAseq data and supporting the subsequent investigation of these neuroinflammatory markers in aged PP mice. Based on downregulation of Glpr2 in adulthood, indicating vascular hyperperfusion (Xie et al. 2018) and the subsequent upregulation of the vascular and inflammatory markers, $L c n-2$, at 9 months, we investigated vascular integrity by validating the gene expression of Kruppel-like factor 4, KIf-4, at 9 and 16 months and observed a 
transient downregulation at 9 months (Table 2). At the same time point, angiogenesis markers such as Cytochrome P450 Family 1 Subfamily B Member 1, Cyp1b1, and Angiopoietin-like 4, Angptl4, with reported function in vascular AD and homeostasis (Chakraborty et al. 2018; Ghosh et al. 2016) showed a peak at 9 months, supporting pathological vascular processes in aged adult PP mice.

Nevertheless, an increase in Lcn2 has been reported in both AD brains (Dekens et al. 2018; Naudé et al. 2012) and CSF from vascular dementia patients (Cerebrospinal Fluid) (Llorens et al. 2020a) raising the possibility that this molecule can capture a mixed vascular-AD pathology. In line with our Polyl:C model of sterile infection, the increase in Lcn-2 is likely attributed by the production and release by activated microglia, reactive astrocytes, neurons, and endothelial cells in response to inflammatory and infectious insults (Jha et al. 2015). Our immunofluorescence analysis using an antibody specific for Lcn2 shows an increase in Lcn2 protein expression in the hippocampal CA3 field of the Polyl:C mice at 9 months and 16 months aged mice (Fig. 6A). At 16 months, small $A \beta 1-42$ aggregates are visible in the PP hippocampus in close association with Lcn2 positive cells (Fig, 6A, insert). Quantitative analysis of the Lcn2 signal shows a significant increase in the \% of the Lcn2 stained area in PP mice in both age groups (Fig. 6B). To study lipid metabolism and intracellular lipid droplets accumulation, as a sign of neuroinflammation with aging, we have utilized the dye Nile Red which accumulates in lipids and emits red fluorescence (Greenspan, Mayer, and Fowler 1985). We observed more Nile Red-positive lipid droplets (LDs) in the hippocampal CA3 field with aging, which is even more evident in PP mice as compared to saline controls (Fig. 6C). Triple labeling with Nile Red, Neurofilament L-200, and Iba1, shows that both NF200 positive neurons and Iba1 positive microglial cells have increased lipid droplets (Fig 6D). Quantitative analysis of the LDs, represented as fold change between the PP and NN at the different time points indicates that the density of the LDs is significantly greater in PP mice starting from 6 months, resulting in increased stained area, peaking at 6 months (Fig. 6E). On the other hand, LDs' size shows a small but significant expansion (30\%) in PP mice at 3months but afterward remains unchanged between conditions (Fig. 6E). The histo-anatomical analysis confirms the presence of neuroinflammatory markers that contribute to an AD-like neuropathological progression.

\section{Translation into Alzheimer's disease}

To assess whether the newly designed Polyl:C model is reproducing genetic changes in human $A D$, we performed targeted fingerprinting focusing in a cross-sectional cohort of post-mortem entorhinal cortices containing the hippocampus from age-matched subjects with mild-moderate $A D$, severe $A D$ and age-matched healthy controls (CTL) (Table 3 and Suppl. Table 1). We first examined cell-type-specific genes, GFAP, Iba1, and MAP2. Consistently, with our model we observe a progressive increase in GFAP expression from Moderate to Severe AD (Fig. 7A and Suppl. Table 8), while Iba1 and MAP2 remain unchanged (Fig. 7A and Suppl. Table 8). Next, we examined some of the relevant DEG with reported association with $A D$ and divided them into functional categories. Glpr2 and Ide belonging to the glucose metabolism with differential expression in a 6 months old PP adult, did not show any significant difference between the clinical groups and controls (Fig. 7A and Suppl. Table 8). We next examined Kcnj2 and Egr2 which show downregulation in the PP model at 9 months (Table 2 and Fig. $5 \mathrm{H}$ ) and observed high variability with no changes across stages (Suppl. Table 8 and Fig 7C). Among the cellular signaling genes, 
with specific repression in the PP model, we detect an opposite increasing trend in c-Fos, c-Jun, and Notch1 to the PP model with a significant 3.1 upregulation of $c$-Fos in severe AD as compared to controls (Suppl. Table 8 and Fig 7D). In the lipid metabolism group, Plin4 and Lcn2 show no change opposite to the PP model (Suppl. Table 8 and Fig 7E), suggesting that those molecules are more implicated in vascular inflammation. To validate this assumption, we analyzed the vascular genes, Klf4, Angpt/4, Cyp1b1, which showed transient alterations in the PP mode, and observed no change across stages (Suppl. Table 8 and Fig 7F). To understand the dependencies between the examined genes, we performed correlation analysis using the aggregate population. In general, all interactions are moderately significant $(r>0.5)$ with positive associations between $c$-fos and GFAP, matching the increasing trend of the two transcripts, which suggest a cell-typespecific change, while $c$-fos is negatively associated with MAP2 (Fig. 7G). Kcnj2 is inversely associated with Ide reflecting their opposite trend, while Ide is positively correlated with Cyp1b1 (Fig. 7G), indicating potential dependencies. Other interactions among the studied genes are seen but too subtle to be of functional relevance. Overall, despite the Polyl:C model replicates some aspects of the AD proteinopathy and microglia changes, the genetic expression between the mouse and humans differs substantially, raising the possibility of a mixed-vascular-AD model with diverse genetic fingerprints.

\section{Translation into vascular dementia}

To verify the hypothesis of a mixed vascular-AD phenotype, we performed gene targets' validation on a second cohort of hippocampi from vascular dementia patients and age-matched healthy controls (Table 4 and Suppl. Table 2). Cell type specific genes, GFAP and Iba1, indicate an increasing but not significant trend of microglia and astroglia, while MAP2 levels remain unchanged. Accordingly to our model, Glpr2 decreases by $90 \%$ in vascular dementia as compared to controls (Fig. 7J and Supplementary Table 9) suggesting ongoing vascular hypoxia ((Xie et al. 2018)). On the other hand, the inflammatory and vascular markers, Lcn2, Cyp1b1, increase in the vascular dementia as in the PP model (Fig. 7L and 7K and Supplementary Table 9). Opposite to the genetic expression in the mouse, KIf4, Notch1 and c-fos levels rise in vascular dementia (Fig. 7M and 7Y and Supplementary Table 9). Correlation analysis of the differentially expressed genes in the aggregate cohort indicates a positive association ( $r>0.6)$ among Lcn2, Notch1 and Klf4, implicating those factors in the vascular pathology. Whereas, along the mechanistic trajectory of vascular remodeling a negative association is observed between Glpr2 and the two angiogenesis genes, KIf4 and Cyp1b1. These results indicate that a handful of the gene targets that are significantly affected upon systemic inflammation in the brain of PP mice are reproduced in vascular neurodegenerative dementia.

\section{Discussion}

\section{Spread of peripheral inflammation to the brain}

$A D$ is a multifactorial complex disorder requiring the understanding of causal and risk factors for proper treatment. Currently, much research has been focused on the amyloid hypothesis. However, the large majority of clinical trials targeting amyloid- $\beta$ have proved ineffective. Furthermore, evidence indicates that elderly people with $A \beta$ accumulation in the brain may still preserve intact cognition questioning the role of amyloid- $\beta$ in $A D$. On the other hand, modification 
of tau protein and its accumulation correlate better with the pathogenesis and topology of $A D$ pathology (Joie et al. 2020). Nevertheless, if tau matches the neurodegenerative process, it remains a consequential event, while the etiology of sporadic AD lies mainly from the interplay of environmental and endogenous factors. There are increasing reports about a link between infectious diseases causing low-grade inflammatory responses over a lifetime and the development of AD with aging. Herpesviruses (Jamieson et al. 1991; Readhead et al. 2018), as well as bacteria such as Chlamydia Pneumoniae, Borrelia Burgdorferi and Porphyromonas Gingivalis (Hammond et al. 2010; Miklossy et al. 2004; Dominy et al. 2019) , are enriched in the brains of $A D$ patients likely playing a role in the disease pathogenesis. Experiments in transgenic mice for APP and Tau animal using viral, Polyl:C and bacterial, Lipopolysaccharides (LPS), aggravate and accelerate the AD-like pathology, supporting a role for infectious agents in triggering neuroinflammation and the proteinopathy (Krstic et al. 2012; Kitazawa et al. 2005). This prompted the development of a sporadic AD model using Polyl:C in naive non-transgenic mice to address whether a systemic sterile infection is a sufficient driver of the AD-pathology. This was first shown by the work of Kristic and colleagues demonstrated that a single prenatal immune challenge with Polyl:C could trigger an AD-like pathology and sustained brain inflammation (Krstic et al. 2012). Nevertheless, later studies showed no signs of long-term changes in central inflammatory responses or microglial morphology changes after a single prenatal immune activation (Giovanoli et al. 2015). Taking into consideration that infections typically occur over a lifetime, including the prenatal stages, we investigated whether systemic immune challenge using Polyl:C during prenatal (Wischhof et al. 2015; Arrode-Brusés and Brusés 2012; Patrich et al. 2016) and postnatal stages can induce a sustained peripheral inflammation with long-term effects on brain function mimicking the progressive pathophysiology of sporadic AD. The current work is the first cross-sectional and multi-modal examination of such a model, which is a prerequisite to mapping dynamic molecular changes occurring in the brain as a result of chronic peripheral inflammation. With the current experimental paradigm, we found that systemic infection with Polyl:C is associated with upregulation of inflammatory humoral factors (MCP-1, IL-6, IL-10, and TNF- $\alpha$ ) in the plasma starting from 3 months age, with a peak at 6 months, which later decline in the aged mice until complete dissipation at 16 months. Interestingly, elevated IL-6 and IL-10 levels in the blood or brain of $A D$ patients have been associated with the severity of cognitive decline and increased ventricular volume (Licastro et al. 2003; Leung et al. 2013) supporting the association between peripheral inflammation and AD. While the innate immune response to Polyl:C is observed at 3 and 6 months of age in the circulation, the adaptive immunity does not show any deviation (data not shown) and there is no observable infiltration of neutrophils, monocytes nor polymorphonuclear cells into the brain at these stages, suggesting preservation of the blood-brain barrier in younger animals until mid-age. Nevertheless, analysis of proinflammatory markers in the brain shows increased levels of IFN- $\gamma$ at 6 and 9 months, while a rise in IL-6 at 6 months, precedes IL-1 $\beta$ increase at 9 months. This indicates that despite no cellular extravasation, cytokines at the earliest stages can spread from the periphery to the brain, causing and likely propagating neuroinflammation. On the other hand, in the oldest cohort, brain inflammation is exhausted, with an interesting inversion of IL-6 in PP mice suggesting immunodepression in the very aged also reflected by low levels of inflammatory cytokines in the circulation. Furthermore, it is known that IL-6 regulates the balance between pro- and antiinflammatory responses depending on the context contributing to metabolic brain function 
(Borovcanin et al. 2017). For instance, IL-6-/- mice develop obesity and insulin resistance indicating that deficiency of IL-6 can lead to metabolic changes (Wallenius et al. 2002). In parallel to the observed rise of neuroinflammation with aging, we also see an increase in microglia and astroglia gene expression at 6 and 9 months, reflecting cell-type-specific changes in these populations (Fig 1).

\section{Progressive proteinopathy}

Along with the peripheral-central inflammatory responses, we observe a rise in hippocampal tau phosphorylation and p-tau/total tau ratio in accord with studies showing that IL-6 treatment induces tau hyperphosphorylation ( $p$-Tau205/tau) in rat embryonic hippocampal neurons (Quintanilla 2004). In the aged Polyl:C mice, the hippocampal CA neurons accumulate phosphorylated tau protein in their axons with dominating astrogliosis. On the other hand, we observe that soluble $A \beta_{1-42}$ increases at 6 months, coinciding with the rise in neuroinflammation and the onset of the tauopathy. Recent reports have indicated that inflammatory cytokines can increase $\beta$-secretase activity in neurons, producing elevated $\mathrm{A} \beta_{1-42}$ (Alasmari et al. 2018; Hur et al. 2020), which is perfectly in line with our model. This supports the use of anti-inflammatory agents at the early stages as a preventive strategy to the proteinopathy as recently reinforced (Hampel et al. 2020). In the aged PP mice, at 16 months, insoluble tangles or amyloid- $\beta$ aggregates are visible in the hippocampus as small neuropil aggregates internalized at times by astroglia cells and in vessels reflecting a CAA. Interestingly, amyloid- $\beta$ deposits in vessels are commonly seen in severe AD patients (53\% of cases) but also in aged cognitively healthy individuals (50\%) (Kövari et al. 2013). In our model, CAA-ike structures were however seen only in PP animals suggesting that the proteinopathy associated with vascular lesions is caused by systemic inflammation only. In the entorhinal cortex, gateway between the neocortex and the hippocampus controlling memory and spatial navigation (Fyhn et al. 2004), old PP mice display fibrillar aggregates. All these progressive changes are associated with working memory deficits displayed as a reduction in alternation at the Y-maze in agreement with the early prenatal and late postnatal Polyl:C mouse model (Krstic et al. 2012). Thus, spatial memory deficits upon Polyl:C challenge may be contributed by the progressive tauopathy in the limbic system accompanied by growing fibrillary aggregates disrupting the neural networks' integrity responsible for spatial memory encoding.

\section{Microglia phenotypic change}

Microglia are the major innate immune cells of the central nervous system mediating host defense responses against infectious agents, injury, abnormal accumulation of amyloid- $\beta$, and prion proteins (Yin et al. 2017). These cells express TLR3 viral receptors recognizing double-stranded RNA viruses (Town et al. 2006) and therefore play an important role in neuroinflammation in response to such stimuli initiating neuronal death. We have reported here a full-length characterization of microglial morphological changes across aging within the hippocampus of Polyl:C mice. Our results indicate dynamic changes in microglia soma features as well as its cell processes. Both pathological and non-pathological insults activate microglia changing from its normal ramified, rounded, and small soma to more hyper-ramified, reactive phagocytic morphology with aging (Walkera, Nilsson, and Jones 2013), which are observed in our model and are accompanied by the dynamic raise in inflammatory cytokines (IL-6, IL-10 and IFN- $\gamma$ ). In 
alignment with our study, others have reported an enlargement in the microglia soma/volume and an increase in their branch points after bacterial LPS exposure (Siemsen et al. 2020). As reviewed in other studies (Knuesel et al. 2014) the effect of second immune challenge postnatally exacerbates the AD-like pathology and the dynamic changes in the microglial morphology indicate a potential priming effect due to both maternal and early postnatal immune activation. Many models have been proposed to understand the morphological dynamic changes, for example after injury (Walker et al. 2014). In our study morphological changes in the aging Polyl:C mice resemble a microglial phenotype upon injury (Streit, Walter, and Pennell 1999) and after acute inflammatory response with neuraminidase treatment (Fernández-Arjona et al. 2017). While recently, it has been pointed out that the genetic repertoire in rodents and human microglia differs substantially (Masuda et al. 2019), the phenotypic transitions of microglia cells in this and other models recapitulates the dynamic undergoing changes in the progression of AD. Our and other findings support that neuroinflammation, passed on by the circulation and in response to the proteinopathy, is perpetuated influencing microglia cell fate to acquire a synapto- and neuro-toxic phenotype (Combs et al. 1999).

\section{Genetic remodeling}

To further support the use of the Polyl:C mouse model as a viable preclinical experimental animal for $A D$ research, we discover alterations in the hippocampal transcriptome relevant to inflammation and neurodegenerative dementia. GEA using the SynGO database indicates the biggest changes in presynaptic gene markers at the early stages (3 months), a steady-state at 6 months, and from 9 months on a progressive enrichment in post-synaptic genes. This is in line with the observed early presynaptic release of glutamate in response to oligomeric Amyloid- $\beta$ (Palop and Mucke 2010), followed in time by post-synaptic scaling events aimed at preserving neuronal integrity at the expense of synaptic transmission (Findley et al. 2019). This mechanistic progression is theoretically confirmed by an aggregate GEA using KEGG, Wikipathway and Reactome pathways, which shows an early abundance in calcium signaling cascades, inflammation pathways, including MAPK signaling, PI3K-AKT signaling associated to cell survival and apoptosis. While at 6 months we confirmed an important decrease in Glpr2 associated to synaptic depotentiation in response to hyperactivity (Sasaki-Hamada, Ikeda, and Oka 2019), (Xie et al. 2018; Bhusal et al. 2019), the changes in gene expression are much pronounced in the midaged 9 months and older 16 months PP mice with a 10\% gene overlap. Longitudinal characterization revealed the upregulation of three genes (Plin4, H2-Aa, Lcn2) related to proinflammatory and glial function and downregulation of three genes (Jun, Egr2, Kcnj2) linked to neuronal or ion transport, reinforcing the mechanisms occurring in the progression of the neuropathology. We confirm a stark increase of Lcn2 and Plin4 at the late stages. Lcn2 is a key gene involved in iron regulation and inflammation (Dekens et al. 2018). It is upregulated during systemic inflammation (Kang et al. 2017) and has been found to be elevated in the brains of AD patients (Naudé et al. 2012). Plin4 is often associated with triacylglycerol metabolism and is involved in the biogenesis of lipid droplets in pathological degeneration (Han et al. 2018). The Polyl:C model shows a strong and specific rise in lipid droplets density accompanied by an increase in Lcn2 protein levels in neurons and glia, which confirms the metabolic and inflammatory disbalance in response to systemic immune challenge. The increase of Lcn2 in neurons and glial cells generates neuroinflammatory responses associated with insulin resistance 
and synaptic modulation (Song and Kim 2018)accumulation in the endothelial barrier (Ferreira et al. 2015) affects BBB permeability, as the genetic profiling in aging PP mice suggest. Thus the multifacet function of $\mathrm{Lcn} 2$ will result in increased neuroinflammation and reactive astrogliosis $(\mathrm{Bi}$ et al. 2013) which is confirmed in PP mice. The sterile-infection driven Lcn-2 supports its role as a putative druggable target to halt the immune-driven neuropathological progression.

\section{Reproducibility in postmortem tissue from AD and vascular dementia patients}

One of the challenges of understanding the physiopathology of $A D$ to develop targetable therapeutics is partially attributed to the poor reproducibility between animal models and humans and multifactorial etiology of the disease(Götz, Bodea, and Goedert 2018). Inflammatory, vascular processes and misfolded proteins should be considered as a whole and investigated closely to unravel dependencies. Furthermore, overlapping pathologies between $A D$ and $\mathrm{VaD}$, representing the most common forms of dementia, are present in more that $20 \%$ of the cases supporting common mechanisms and therapeutic investigations. Consistently, our study indicates that the PP model shows an AD proteinopathy coupled with vascular deficit. This conclusion derives from our DEG target repertoire analysis in two cohorts representing i) progressive AD stages and ii) vascular dementia. We confirm an astrogliosis in AD, captured by increased GFAP levels, comparable to the PP model while this effect is present but less pronounced in VaD. Astrogliosis has been shown to increase linearly with cognitive decline and is strongly associated with plaques and tangle formation (Serrano-Pozo et al. 2011). On the other hand in VaD, astrogliosis and astroglial endfeet swelling has been implicated as one of the triggering factors for vascular damage (Price et al. 2018; Wang et al. 2018). In both instances, reactive astrogliosis is a commonality of the two diseases associated with the production of pro-inflammatory cytokines such as IL-6, which is strongly upregulated in the PP model at 6 and 9 months. Along with the increase in GFAP expression, we see a positive association with $c$-fos and Notch1 levels in the severe AD stage, supporting the proliferation of astroglia (Hisanaga et al. 1990) and the role of Notch1 in driving astroglia proliferation in response to inflammation through the proto-oncogene c-fos (Acaz-Fonseca et al. 2019). This data is in opposition to the PP mouse model, where despite an increase in astroglia, a decline in c-fos and Notch1 is observed at 9 months. The discrepancy can be explained by the different signaling profiles of glia and neurons in rodents, suggesting that cellular cascades may be cell-specific depending on the species.

Investigating the genes involved in mediating vascular function (Deniz, Bozkurt, and Kurtel 2007), we observe no change in Glpr2 expression in the progression of $A D$, while in VaD, Glpr2 is downregulated similarly to the PP model supporting that cognitive deficit is contributed by lower blood perfusion ((Xie et al. 2018)). Along the same lines, the progressive increase in the inflammatory and metabolic markers, Lcn2, displayed by aging PP animals, is not reproduced in $\mathrm{AD}$ but in the $\mathrm{VaD}$ specimen. This finding is in contrast to the previous reports of a rise in Lcn2 protein levels in the hippocampus of severe AD subjects (Naudé et al. 2012) and patients with $\mathrm{MCl}$ (Choi, Lee, and Suk 2011), but is completely aligned with the recent reported upregulation of Lcn2 in CSF from VaD (n.d.; Llorens et al. 2020b). In support of the microvessel damage mediated by Lcn2 (J.-H. Kim et al. 2017), 9 months old PP mice report a rise in Cyp1b1, Angpt/4 and reduction in Klf4, which all regulate the BB permeability (Sangwung et al. 2017; Palenski et al. 2013; Huang et al. 2011). While in AD those markers remain unchanged, in VaD Cyp1b1 is increased together with Klf4, while Angpt/4 remains unchanged. The different directionality of 
those vascular markers between mouse and human can be explained either by the time point of sampling or the species diversity. Nevertheless, a vascular pathology in the PP model is supported by two-fold reduction of Claudin 5, Cldn5, a key regulator of BBB permeability. While mixed pathologies in neurodegenerative diseases are not uncommon accounting for more than $20 \%$ of the cases (Custodio et al. 2017), the PP model shows a mixed pathology with pronounced neuroinflammation, AD-like proteinopathy, and vascular factors. Interestingly, a recent finding has shown that hyperphosphorylated Tau affects neurovascular coupling (Park et al. 2020) bridging AD-mechanism to the vascular deficit. We acknowledge that the number of human samples analyzed is small, however the translational validation of the Polyl:C data clearly demonstrates that this mouse model can reproduce some of the characteristic features of reactive central inflammation (Hampel et al. 2020) and vascular pathology encountered in neurodegenerative dementia.

\section{Conclusions}

The present research, using the Polyl:C mouse model of sterile infection, demonstrates that chronic systemic inflammation during adulthood causes a progressive neuropathology with neuroinflammation, insoluble protein aggregates, vascular permeability, microglia remodeling and behavioral deficits, mimicking a mixed vascular-AD pathology. While the Polyl:C model has been previously employed in the prenatal stage or in old age, the present longitudinal study adds to the understanding of the effects of sustained inflammation on brain homeostasis over a lifetime. Our post-mortem analysis on $\mathrm{AD}$ and $\mathrm{VaD}$ brain specimens, shows partially overlapping genetic profiles between $\mathrm{VaD}$ and the Polyl:C mouse supporting that systemic inflammation can cause vascular deficit besides neuroinflammation and proteinopathy. Indeed, chronic inflammation is known to pose a risk for cardiovascular health which with aging may contribute to overlapping pathologies (Metti and Cauley 2012; Newcombe et al. 2018). The study is of use not only for the understanding of the interplay between peripheral and central processes but also presents a surrogate animal model displaying a sporadic vascular AD-mixed phenotype, which can be used for testing therapeutics against pathological brain aging.

\section{List of Abbreviations}

$\begin{array}{ll}\text { ABCA7 } & \text { ATP-binding cassette sub-family A member 7 } \\ \text { AD } & \text { Alzheimer's disease } \\ \text { Angptl4 } & \text { Angiopoietin like } 4 \\ \text { ApoE } & \text { Apolipoprotein E } \\ \text { ApoE } \varepsilon 4 & \text { Apolipoprotein E variant } \varepsilon 4 \\ \text { A } 3 & \text { Amyloid beta } \\ \text { BBB } & \text { Blood Brain Barrier } \\ \text { BIN1 } & \text { Box-dependent-interacting protein } 1 \\ \text { CA } & \text { Cornu Ammonis } \\ \text { CAA } & \text { Cerebral Amyloid Angiopathy }\end{array}$


CD2AP

CD33

CELF1

c-fos

Cyp1b1

Cldn5

CLU

CNS

CR1

CSF

CTL

Cx

DABCO

DAPI

DEGs

dsRNA

EC

EDTA

EGR2

EPHA1

FERMT2

GD

GEA

GFAP

GlpR2

GO

GRIN1

GWAS

$\mathrm{H} 2-\mathrm{Aa}$

Iba-1

Ide

IL

INPP5D

KCNJ2

KEGG

KIf4

KS
CD2 Associated Protein

Myeloid Cell Surface Antigen CD3

CUGBP Elav-like family member 1

Proto-oncogene c-Fos

Cytochrome P450 1B1

Claudin 5

Clusterin

Central Nervous System

Complement receptor type 1

Cerebrospinal fluid

Control

Cortex

1,4-diazabicyclo [2.2.2] octane

4',6-diamidino-2-phenylindole

Differentially Expressed Genes

Double-strand RNA

Entorhinal cortex

Ethylenediaminetetraacetic acid

Early growth response protein 2

Ephrin type-A receptor 1

Fermitin family homolog 2

Gestational Day

Gene enrichment analysis

Glial fibrillary acidic protein

Glucagon like peptide 2 receptor

Gene ontology

Glutamate lonotropic Receptor NMDA Type Subunit 1

Genome Wide Association Studies

$\mathrm{H}-2$ class II histocompatibility antigen, A-B alpha chain

Ionized calcium binding adaptor molecule1

Insulin degrading enzyme

Interleukin

Phosphatidylinositol 3,4,5-trisphosphate 5-phosphatase 1

Potassium Inwardly Rectifying Channel Subfamily J Member 2

Kyoto Encyclopedia of Genes and Genomes

zinc finger-containing Krüppel-like factor

Kolmogorov-Smirnov 


\begin{tabular}{|c|c|}
\hline C-Jun & Transcription factor AP-1 \\
\hline LCN2 & Lipocalin 2 \\
\hline LPS & Lipopolysaccharide \\
\hline MAP2 & Microtubule-associated protein 2 \\
\hline MAPK & Mitogen-activated protein kinase 1 \\
\hline $\mathrm{MCl}$ & Mild Cognitively Impairment \\
\hline MCP-1 & Monocyte Chemoattractant Protein-1 \\
\hline MEF2C & Myocyte-specific enhancer factor $2 \mathrm{C}$ \\
\hline $\mathrm{mM}$ & milliMolar \\
\hline M-MLV & Moloney Murine Leukemia Virus Reverse Transcriptase \\
\hline mo & month \\
\hline mRNA & Messenger RNA \\
\hline MS4A4A & Membrane-spanning 4-domains subfamily A member 4A \\
\hline ND & Neurodegenerative disease \\
\hline NME8 & Thioredoxin domain-containing protein 3 \\
\hline NN & Saline controls \\
\hline Notch1 & Neurogenic locus notch homolog protein 1 \\
\hline PFA & Paraformaldehyde \\
\hline PICALM & Phosphatidylinositol-binding clathrin assembly protein \\
\hline PLCG2 & 1-phosphatidylinositol 4,5-bisphosphate phosphodiesterase gamma-2 \\
\hline pM & picomolar \\
\hline PLIN4 & Perilipin 4 \\
\hline PMNs & Polymorphonuclear neutrophils \\
\hline Polyl:C & Polyinosinic:Polycytidylic acid \\
\hline PSEN & Presenilin \\
\hline PP & Polyl:C injected animals \\
\hline pTau & Phosphorylated tau \\
\hline PVA & Polyvinyl alcohol \\
\hline rpm & revolutions per minute \\
\hline rRNA & ribosomal RNA \\
\hline r & Spearman's Rank Correlation \\
\hline SEM & Standard Error Mean \\
\hline SLC24A4 & Sodium/potassium/calcium exchanger 4 \\
\hline SORL1 & Sortilin-related receptor \\
\hline SRCAP & Snf2-related CREBBP activator protein \\
\hline TBS & Trizma-based salt solution \\
\hline TLRs & Toll Like receptors \\
\hline
\end{tabular}




$\begin{array}{ll}\text { TNFa } & \text { Tumor necrosis factor alpha } \\ \text { TREM2 } & \text { Triggering receptor expressed on myeloid cells 2 } \\ \text { ZCWPW1 } & \text { Zinc finger CW-type PWWP domain protein 1 } \\ \mu \mathrm{g} & \text { microgram } \\ \mu \mathrm{m} & \text { micrometer }\end{array}$

\section{Declarations}

\section{Ethical Approval and Consent to participate}

Animal experimentation was approved by the animal experiment committee, University of Fribourg (Protocol no. 2016_32_FR registered 01/01/2017).

The use of human tissue has been approved by the Ethical Commission of the Brain Bank for Dementia UK (OBB443 registered 1/05/2017 and OB344 registered 1/02/2014), Stanford (Stanford IRB), and the Ethical Commission from the Canton of Fribourg and Vaud (N. 325/14). All experiments conducted on human tissue comply with the WMA Declaration of Helsinki.

\section{Consent for publication}

All authors agree on publishing the original data

\section{Availability of supporting data}

Supporting data is available in the form of supplementary material and tables. All raw data is available on request.

\section{Competing interests}

There are no competing interests

\section{Funding}

Schweizerischer Nationalfonds zur Förderung der Wissenschaftlichen Forschung (163470)(LA). Bundesbehörden der Schweizerischen Eidgenossenschaft (2017.0480) (PB).

\section{Authors' contributions}

PB conducted the main bulk of the experiments and contributed to the writing. ID performed the bioinformatic analysis. EZ and ET performed the cellular immunology experiments from blood and brain. AF and EB performed the blind quantitative analysis of microglia morphology using custom-made scripts. MAD assisted in the inflammatory model characterization. LA designed the study and wrote the manuscript. All authors read and approved the final manuscript

\section{Acknowledgments}

We would like to thank Mrs. E. Martin and V. Tache for the technical support. We are also grateful to Dr. M. Reggente in providing his expertise in R. We thank Prof. T. Montine for 
sharing the brain tissue from the Stanford Biobank for this study. We are thankful to the donors and their families for allowing us access to the human tissue (Oxford, UK and Stanford, USA).

\section{Figure Legends}

Figure 1. The neuroinflammatory evolution in aging Polyl:C mice. (A) Timeline of the experimental procedure and Polyl:C injections. (B) RT-PCR analysis of proinflammatory cytokines in the hippocampus after the Polyl:C treatment. Transcript analysis for (C) neuronal (GRIN1), (D) microglia (Iba1), and (E) astroglial (GFAP) markers. (F) The correlation matrix indicates the associations between time series of Grin1, Iba1, GFAP with the examined cytokines. Values represented as mean $\pm S E M, n=4-5$ mice per age, and treatment. GD=gestational day, $B D=$ birthday. ${ }^{*} p<0.05,{ }^{* *} p<0.005, \# p=0.07$. Student's t-test.

Figure 2. The Effect of Polyl:C on p-tau accumulation in the hippocampus. (A) Immunoblots of hippocampal lysate from PP and NN mice at 3, 6, 9, and 16 months, for p-tau, tau, GFAP, Synaptophysin. $\beta$-Actin was used as housekeeping control. (B, D) Relative quantification of ptau, tau, GFAP, and Synaptophysin represented in normalized optical density. (C, E) Percentage of relative changes of these proteins compared to the saline treated controls (NN). Representative immunostaining for (F) p-tau, Thioflavin $S$ (9 months), and (G) $A \beta_{1-42}$, p-tau, and GFAP at 16 months age group. Scale bar in $\mathrm{H}$ is $45 \mu \mathrm{m}$, $\mathrm{J}$ is $20 \mu \mathrm{m}$. Values represented as mean $\pm \mathrm{SEM}, \mathrm{n}=4$ 5 mice per age, and treatment. ${ }^{*} p<0.05,{ }^{* *} p<0.005, \# p=0.07$. Student's t-test

Figure 3. Amyloid fibrils in the hippocampus and entorhinal cortex with spatial memory deficits. (A) Double immunolabeling for $A \beta_{1-42}$ and GFAP shows internalization of amyloid in GFAP + glia cells (stars) in NN and PP mice at 16 months and strong depositions of amyloid in vessels (white arrows) in PP mice. (B) Representative immunostaining of $A \beta_{1-42}$ and $p T a u^{231}$ in the hippocampus and (C) Entorhinal cortex of aged 16 months PP \& NN controls. Insert showing p-Tau \& A $\beta_{1-42}$ colocalization. (D) Quantification of amyloid aggregates $>5 \mu m^{2}$ and (E) percentage area covered per region of interest $(n=5-6$ mice per condition \& 4-5 ROI per mice). (F) Immunobead assay showing dynamic trend in soluble $A \beta_{42}$ level fold changes in the cortical lysates relative to control ( $n=4-6$ mice per age group \& condition). (G) Box plots indicate a significant impairment in the percent of spontaneous alternation entry in PP mice compared from 6 to 16 months as compared to NN ( $n=7-10$ mice per treatment and age). Values in bar charts and line graph are represented as mean \pm SEM. ${ }^{*} p<0.05$, ${ }^{* *} p<0.005$ Student's t-test. Scale bar in $A$ and $B=20 \mu m$.

Figure 4: Longitudinal changes in hippocampal microglia. (A) Immunofluorescence staining for Iba1 and $A \beta$ staining in the Polyl:C treated mice. (B) Dynamic changes in microglia soma size, perimeter and circularity were quantified in Polyl:C (PP) mice in different age groups. Data represented as $\%$ relative to controls. ( $n=150-180$ cells from 3-4 mice per group \& condition in $3 \mathrm{~m}, 6 \mathrm{~m}$ stages; $n=85$ cells in $9 \mathrm{~m}$ and $n=55$ cells at 16 months from 3 mice). (C) Bar chart of \% relative values relative to controls for the average endpoints, maximum branch length, and branch length per cell. Red dotted line indicates the 100 percent control line ( $n=55-65$ ROIs/3, 6,9 months group and 25 ROls/16 months; $n=3$ mice per age group and condition). (D) Example of fractal 
analysis of microglia from the hippocampus of Polyl:C and saline treated 16 months aged mice. Single-cell fractal analysis indicates the surface area $(\mathbf{E})$ and perimeter $(\mathbf{F})$ is decreased in PP mice, in contrast, the span ratio $(G)$ showed a higher trend. PP mice showed a significant decrease in circularity $(\mathbf{H})$ and fractal dimension (I) $(n=45$ cells from 3 mice per group and condition). Values in the bar charts are represented as mean \pm SEM. $\# p=0.07,{ }^{*} p<0.05,{ }^{* *} p<0.005$ Student's t-test. Scale bar, $A=20 \mu \mathrm{m}$.

Figure 5: Bulk transcriptomics analysis of the hippocampus in the aging Polyl:C. Gene expression changes after the prenatal and early postnatal immune challenge. Volcano plot with log2-fold change (X-axis) and -log10 p-value (Y-axis) at different age groups (A) 3 months (B) 6 months (C) 9 months and (D) 16 months in PP versus NN controls. Genes with log2 fold changes $>0.5$ are shown in green. Significantly regulated genes are shown in red (log2 fold change $>0.5$, $\mathrm{p}<0.05$, left downregulated, and right upregulated). Genes with insignificant log2 fold changes $<0.5$ are shown in black [controls (NN) $n=3 /$ age, Polyl:C (PP) $n=3 /$ age]. (E) Venn diagram indicating the number of uniquely and commonly affected genes in the aging hippocampus following prenatal and early postnatal Polyl:C (PP) treatment. Few common genes along with their Log2 Fold changes either (F) downregulated or (G) upregulated were plotted across staging. Transcript validation analysis for (H) Egr2 and Kcnj2 and (I) Plin4 and Lcn2. (J) Correlation matrix with highlighted significant association from 9 to 16 months among the differentially expressed cell-type markers (GFAP, Iba1, and Grin1) and Egr2, Kcnj2, Plin4 and Lcn2 between Polyl:C and saline controls [controls (NN), $n=5 /$ age, Polyl:C (PP) $n=4-5 /$ age]. Line plots show values as mean \pm SEM. ${ }^{*}=p<0.05,{ }^{* *}=p<0.01, \#=p @ 0.1$, Student's t-test.

Figure 6: Effect of Polyl:C on the hippocampal Len2 \& lipid droplet density. (A) Representative Lcn2, $A \beta_{1-42}$ stained confocal images of saline and Polyl:C treated mice in 9 months and 16 months aged PP mice. Larger aggregates of Lcn2 (insert) visible at the 16 months Polyl:C exposed mice. (B) Quantification of \% stained area indicates increased Lcn2 expression in the hippocampal CA3 field ( $n=4-6$ mice for 9- and 16-months groups/treatment). (C) Nile red positive lipid droplet staining (violet) across aging in NN \& PP mice. (D) Representative triple fluorescence labeling with Nile Red, Iba1 (teal) and NF200 (grey) shows an overlap between the different marker localized lipid droplets in Iba1 positive cells. Arrows indicating NileRed ${ }^{+} / \mathrm{lba1}^{+}$ cells. (E) Bar chart representing fold change values in the lipid droplet density, mean size, and \% stained area per ROI measured. Data are represented as mean $\pm S E M$ relative to control $(n=3-4$ mice per group/treatment). ${ }^{*} p<0.05,{ }^{* *} p<0.005$, Student's t-test;. Scale bar $A=20 \mu m, C, E=10 \mu m$.

Figure 7. Target transcripts validation in the human brain specimen of patients with AD and $\mathrm{VaD}$. Bar plots with Jitter showing the fold changes (FC) of the DEGs (A-F) in moderate AD and severe $A D$ entorhinal cortices as compared to healthy controls (CTRL) and $(\mathbf{H}-\mathbf{N})$ in hippocampal sections from $\mathrm{VaD}$ as compared to healthy controls (CTRL). (A and $\mathbf{H}$ ) FC of celltype specific markers for astroglia (GFAP), microglia (lba1) and neurons (MAP2). (B and I) FC of glucose metabolism markers, Glpr2 and Ide. (C and J) FC of plasticity markers, Egr2 and Kcnj2. (D and K) FC of cell signaling markers, c-fos, c-Jun and Notch1. (E and L) FC of inflammatory and metabolic markers, Lcn2 and Plin4. (F and M). FC of vascular markers, Angpt/4, Cyp1b1 and KIf4. (G and N) Correlation matrix of the selected biomarkers in the two study cohorts. Data are 
represented as Geometric mean of fold change \pm Geometric SEM relative to healthy controls. $A D=$ Alzheimer's disease and $\mathrm{VaD}=$ vascular dementia.

\section{Tables}

Table 1: Plasma inflammatory panel in aging PP and NN mice

\begin{tabular}{|c|c|c|c|c|c|}
\hline \multirow{3}{*}{ Age } & \multirow{2}{*}{ Group } & $\begin{array}{c}\text { Chemokine } \\
\text { (pg/ml) }\end{array}$ & \multicolumn{3}{|c|}{ Inflammatory cytokines } \\
& & MCP-1 & IL-6 & TNF-From & IL-10 \\
\cline { 2 - 6 } & NN & $11.1 \pm 0.7$ & $7.1 \pm 0.2$ & $3.8 \pm 0.3$ & $2.7 \pm 0.2$ \\
\hline \multirow{3}{*}{3 months } & PP & $19.8 \pm 1.9$ & $8.3 \pm 0.3$ & $3.3 \pm 0.2$ & $7.7 \pm 0.7$ \\
\cline { 2 - 6 } & Fold change & $1.8^{*}$ & 1.2 & 0.9 & $2.9^{*}$ \\
\hline \multirow{3}{*}{6 months } & NN & $12.1 \pm 3.4$ & $2.4 \pm 0.2$ & $3.2 \pm 0.2$ & $4.1 \pm 1.6$ \\
\cline { 2 - 6 } & PP & $29.8 \pm 11.9$ & $15.8 \pm 10.6$ & $6.8 \pm 0.7$ & $12.0 \pm 3.6$ \\
\cline { 2 - 6 } & Fold change & $2.5^{*}$ & $6.5^{* *}$ & $2.1^{* *}$ & $2.9^{* *}$ \\
\hline \multirow{3}{*}{9 months } & NN & $35.7 \pm 26.9$ & $243.4 \pm 187.5$ & $8.9 \pm 0.7$ & $16.4 \pm 4.7$ \\
\cline { 2 - 6 } & PP & $17.2 \pm 8.4$ & $1464.2 \pm 184.5$ & $8.3 \pm 0.2$ & $9.1 \pm 1.9$ \\
\cline { 2 - 6 } & Fold change & 0.5 & $6.1^{* *}$ & 0.9 & 0.6 \\
\hline \multirow{3}{*}{16 months } & NN & $2.5 \pm 1.0$ & $6.5 \pm 0.1$ & $8.0 \pm 0.3$ & $10.5 \pm 2.1$ \\
\cline { 2 - 6 } & PP & $2.2 \pm 0.5$ & $6.9 \pm 0.3$ & $7.9 \pm 0.2$ & $4.7 \pm 1.7$ \\
\cline { 2 - 6 } & Fold change & 0.9 & 1.1 & 1.0 & 0.5 \\
\hline
\end{tabular}

Data are represented as mean \pm SEM. $N N=$ prenatal\& postnatal saline injected controls, $\mathrm{PP}=$ prenatal and postnatal Polyl: $C$ treated mice. 3 months $(\mathrm{NN}=4, \mathrm{PP}=4), 6$ months $(\mathrm{NN}=8, \mathrm{PP}=8), 9 \&$ 16 months (NN, PP=5 each). ${ }^{*} p<0.05,{ }^{* *} p<0.01$ versus control group, Student's t-test

Table 2 Validation of transcripts via qPCR in aging PP mice as compared to age-matched NN

\begin{tabular}{|c|c|c|c|c|c|c|}
\hline Age & Gene & $\log 2 F C$ & $\mathrm{FC}$ & Regulation & Pathways\& Functions & $P$ value \\
\hline \multirow{5}{*}{3 months } & $\operatorname{Lcn} 2^{*}$ & & -0.32 & Down & $\begin{array}{l}\text { Inflammatory signaling; } \\
\text { dendritic spine pruning }\end{array}$ & 0.02 \\
\hline & Kcnj2* & & 1.01 & - & $\begin{array}{l}\text { Ionic conductance; slow } \\
\text { inhibitory current }\end{array}$ & 0.95 \\
\hline & Egr2* & $-0.1(\mathrm{NS})$ & 0.9 & - & Early immediate gene & 0.94 \\
\hline & Plin4* & 0.6 (NS) & 3.1 & - & Lipid metabolism & 0.23 \\
\hline & Cacna1g & -0.6 & 0.9 & - & Calcium channel & 0.77 \\
\hline \multirow{5}{*}{6 months } & Len2 & & -0.6 & - & $\begin{array}{l}\text { Inflammatory signaling; } \\
\text { dendritic spine pruning }\end{array}$ & 0.70 \\
\hline & Glpr2 & -3.7 & -0.2 & Down & $\begin{array}{l}\text { Akt-mTOR; synaptic } \\
\text { activity, neuroprotection }\end{array}$ & 0.03 \\
\hline & lde & 0.5 & 1.6 & - & $\begin{array}{c}\text { Insulin signaling; } \\
\text { Amyloid- } \beta \text { processing }\end{array}$ & 0.16 \\
\hline & Kcnj2* & 0.1 (NS) & 1.8 & Up & $\begin{array}{c}\text { Ionic conductance; slow } \\
\text { inhibitory current }\end{array}$ & 0.04 \\
\hline & Plin4* & $\begin{array}{l}-0 . .23 \\
\text { (NS) }\end{array}$ & 0.28 & - & Lipid metabolism & 0.49 \\
\hline 9 months & c-Jun & -0.9 & -1.8 & Down & JNK3 pathway; IEG & 0.05 \\
\hline
\end{tabular}




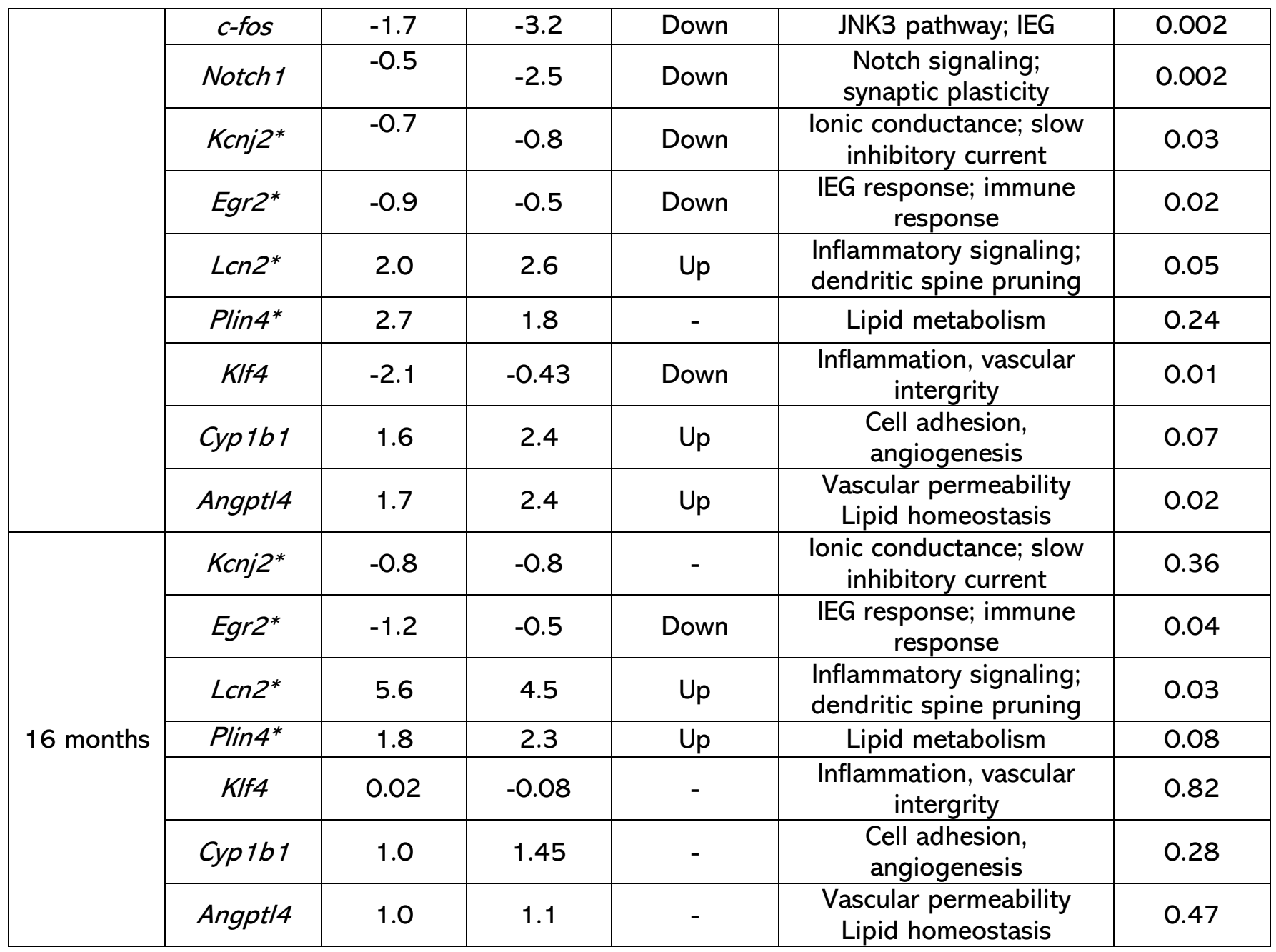

FC=Fold Change; NS=non-significant DEG; * Shared genes with progressive changes in the PP versu NN

Table 3. Summary of Patients' cohort I

\begin{tabular}{|l|c|c|c|c|}
\hline Stage & N. patients & F: $M$ & Age (mean \pm SD) & Braak (mean \pm SD) \\
\hline CTL & 9 & $5: 4$ & $81 \pm 8.8$ & $1.0 \pm 0.5$ \\
\hline MOD & 5 & $3: 2$ & $85 \pm 2.2$ & $4.0 \pm 1.6$ \\
\hline AD & 10 & $4: 6$ & $82 \pm 9.9$ & $5.6 \pm 0.5$ \\
\hline
\end{tabular}

$C T L=$ healthy controls; $M O D=$ moderate $A D ; A D=$ severe $A D$

Table 4. Summary of Patients' cohort II

\begin{tabular}{|l|c|c|c|c|}
\hline Stage & N. patients & F: $M$ & Age (mean \pm SD) & Braak (mean \pm SD) \\
\hline $\mathrm{CTL}$ & 5 & $2: 3$ & $80 \pm 5.9$ & $2.3 \pm 0.5$ \\
\hline $\mathrm{VaD}$ & 6 & $3: 3$ & $82 \pm 3.5$ & $4.0 \pm 1.6$ \\
\hline
\end{tabular}

$\mathrm{CTL}=$ healthy controls; $\mathrm{VaD}=$ Vascular dementia

\section{Bibliography}

Acaz-Fonseca, Estefania, Ana Ortiz-Rodriguez, Iñigo Azcoitia, Luis M. Garcia-Segura, and MariaAngeles Arevalo. 2019. "Notch Signaling in Astrocytes Mediates Their Morphological Response to an Inflammatory Challenge." Cell Death Discovery 5 (April): 85. 
Alasmari, Fawaz, Musaad A. Alshammari, Abdullah F. Alasmari, Wael A. Alanazi, and Khalid Alhazzani. 2018. "Neuroinflammatory Cytokines Induce Amyloid Beta Neurotoxicity through Modulating Amyloid Precursor Protein Levels/Metabolism." BioMed Research International 2018 (October): 3087475.

Alonso, A. C., I. Grundke-Iqbal, and K. Iqbal. 1996. “Alzheimer's Disease Hyperphosphorylated Tau Sequesters Normal Tau into Tangles of Filaments and Disassembles Microtubules." Nature Medicine 2 (7): 783-87.

Arrode-Brusés, Géraldine, and Juan L. Brusés. 2012. "Maternal Immune Activation by poly(I:C) Induces Expression of Cytokines IL-1 $\beta$ and IL-13, Chemokine MCP-1 and Colony Stimulating Factor VEGF in Fetal Mouse Brain." Journal of Neuroinflammation. https://doi.org/10.1186/1742-2094-9-83.

Barber, Robert C. 2012. “The Genetics of Alzheimer's Disease.” Scientifica 2012 (December). https://doi.org/10.6064/2012/246210.

Bhusal, Anup, Md Habibur Rahman, In-Kyu Lee, and Kyoungho Suk. 2019. "Role of Hippocampal Lipocalin-2 in Experimental Diabetic Encephalopathy." Frontiers in Endocrinology 10. https://doi.org/10.3389/fendo.2019.00025.

Bi, Fangfang, Cao Huang, Jianbin Tong, Guang Qiu, Bo Huang, Qinxue Wu, Fang Li, et al. 2013. "Reactive Astrocytes Secrete lcn2 to Promote Neuron Death." Proceedings of the National Academy of Sciences of the United States of America 110 (10): 4069-74.

Bilbo, Staci D., Carina L. Block, Jessica L. Bolton, Richa Hanamsagar, and Phuong K. Tran. 2018. "Beyond Infection - Maternal Immune Activation by Environmental Factors, Microglial Development, and Relevance for Autism Spectrum Disorders.” Experimental Neurology 299 (Pt A): 241-51.

Binda, Anna, Ilaria Rivolta, Chiara Villa, Elisa Chisci, Massimiliano Beghi, Cesare M. Cornaggia, Roberto Giovannoni, and Romina Combi. 2018. "A Novel KCNJ2 Mutation Identified in an Autistic Proband Affects the Single Channel Properties of Kir2.1." Frontiers in Cellular Neuroscience. https://doi.org/10.3389/fncel.2018.00076.

Bindea, Gabriela, Bernhard Mlecnik, Hubert Hackl, Pornpimol Charoentong, Marie Tosolini, Amos Kirilovsky, Wolf-Herman Fridman, Franck Pagès, Zlatko Trajanoski, and Jérôme Galon. 2009. "ClueGO: A Cytoscape Plug-in to Decipher Functionally Grouped Gene Ontology and Pathway Annotation Networks." Bioinformatics 25 (8): 1091-93.

Borovcanin, Milica Milovan, Ivan Jovanovic, Gordana Radosavljevic, Jelena Pantic, Slavica Minic Janicijevic, Nebojsa Arsenijevic, and Miodrag L. Lukic. 2017. "Interleukin-6 in Schizophrenia-Is There a Therapeutic Relevance?" Frontiers in Psychiatry/ Frontiers Research Foundation 8. https://doi.org/10.3389/fpsyt.2017.00221.

Brai, Emanuele, and Lavinia Alberi. 2015. "Simple and Computer-Assisted Olfactory Testing for Mice." Journal of Visualized Experiments: JoVE, no. 100 (June): e52944.

Brai, Emanuele, Swananda Marathe, Simone Astori, Naila Ben Fredj, Elisabeth Perry, Christophe Lamy, Alessandra Scotti, and Lavinia Alberi. 2015. "Notch1 Regulates Hippocampal Plasticity Through Interaction with the Reelin Pathway, Glutamatergic Transmission and CREB Signaling." Frontiers in Cellular Neuroscience 9 (November): 447.

Chakraborty, Ananya, Alwin Kamermans, Bert van het Hof, Kitty Castricum, Ed Aanhane, Jack van Horssen, Victor L. Thijssen, et al. 2018. "Angiopoietin like-4 as a Novel Vascular Mediator in Capillary Cerebral Amyloid Angiopathy.” Brain. https://doi.org/10.1093/brain/awy274.

Choi, Jihye, Ho-Won Lee, and Kyoungho Suk. 2011. "Increased Plasma Levels of Lipocalin 2 in Mild Cognitive Impairment.” Journal of the Neurological Sciences 305 (1-2): 28-33.

Combs, Colin K., Derrick E. Johnson, Steve B. Cannady, Timothy M. Lehman, and Gary E. Landreth. 1999. "Identification of Microglial Signal Transduction Pathways Mediating a Neurotoxic Response to Amyloidogenic Fragments of $\beta$-Amyloid and Prion Proteins." The Journal of Neuroscience: The Official Journal of the Society for Neuroscience 19 (3): 928-39.

Crawley, Jacqueline, and Kathleen Bailey. 2008. "Anxiety-Related Behaviors in Mice." Methods of Behavior Analysis in Neuroscience, Second Edition. https://doi.org/10.1201/noe1420052343.ch5. 
Cunningham, Colm. 2013. "Microglia and Neurodegeneration: The Role of Systemic Inflammation." Glia 61 (1): 71-90.

Cunningham, Colm, and Edel Hennessy. 2015. "Co-Morbidity and Systemic Inflammation as Drivers of Cognitive Decline: New Experimental Models Adopting a Broader Paradigm in Dementia Research." Alzheimer's Research \& Therapy 7 (1): 1-13.

Custodio, Nilton, Rosa Montesinos, David Lira, Eder Herrera-Pérez, Yadira Bardales, and Lucía Valeriano-Lorenzo. 2017. "Mixed Dementia: A Review of the Evidence." Dementia \& Neuropsychologia 11 (4): 364-70.

Davis, Benjamin M., Manual Salinas-Navarro, M. Francesca Cordeiro, Lieve Moons, and Lies De Groef. 2017. "Characterizing Microglia Activation: A Spatial Statistics Approach to Maximize Information Extraction." Scientific Reports 7 (1): 1576.

Dekens, Doortje W., Peter P. De Deyn, Friederike Sap, Ulrich L. M. Eisel, and Petrus J. W. Naudé. 2020. "Iron Chelators Inhibit Amyloid- $\beta$-Induced Production of Lipocalin 2 in Cultured Astrocytes." Neurochemistry International 132 (January): 104607.

Dekens, Doortje W., Petrus J. W. Naudé, Jan N. Keijser, Ate S. Boerema, Peter P. De Deyn, and Ulrich L. M. Eisel. 2018. "Lipocalin 2 Contributes to Brain Iron Dysregulation but Does Not Affect Cognition, Plaque Load, and Glial Activation in the J20 Alzheimer Mouse Model." Journal of Neuroinflammation 15 (1): 330.

Deniz, Mustafa, Ayhan Bozkurt, and Hizir Kurtel. 2007. "Mediators of Glucagon-like Peptide 2-Induced Blood Flow: Responses in Different Vascular Sites." Regulatory Peptides 142 (1-2): 7-15.

Dominy, Stephen S., Casey Lynch, Florian Ermini, Malgorzata Benedyk, Agata Marczyk, Andrei Konradi, Mai Nguyen, et al. 2019. "Porphyromonas Gingivalis in Alzheimer's Disease Brains: Evidence for Disease Causation and Treatment with Small-Molecule Inhibitors." Science Advances 5 (1): eaau3333.

Du, Yanjiao, Chao Liu, Congmin Ma, Xiaohui Xu, Xufeng Zhou, Haitao Zhou, and Chao Huang. 2019. "Cerebral Amyloid Angiopathy-Related Inflammation: A Case Report Presenting with a Rare Variant in SORL1 Gene." BMC Neurology 19 (1): 97.

Edwards, George A., III, Nazaret Gamez, Gabriel Escobedo Jr., Olivia Calderon, and Ines MorenoGonzalez. 2019. "Modifiable Risk Factors for Alzheimer's Disease.” Frontiers in Aging Neuroscience 11. https://doi.org/10.3389/fnagi.2019.00146.

Engelhart, Marianne J., Mirjam I. Geerlings, John Meijer, Amanda Kiliaan, Annemieke Ruitenberg, John C. van Swieten, Theo Stijnen, Albert Hofman, Jacqueline C. M. Witteman, and Monique M. B. Breteler. 2004. "Inflammatory Proteins in Plasma and the Risk of Dementia: The Rotterdam Study." Archives of Neurology 61 (5): 668-72.

Fernández-Arjona, María del Mar, María del Mar Fernández-Arjona, Jesús M. Grondona, Pablo Granados-Durán, Pedro Fernández-Llebrez, and María D. López-Ávalos. 2017. "Microglia Morphological Categorization in a Rat Model of Neuroinflammation by Hierarchical Cluster and Principal Components Analysis." Frontiers in Cellular Neuroscience. https://doi.org/10.3389/fncel.2017.00235.

Ferreira, Ana C., Sandro Dá Mesquita, João C. Sousa, Margarida Correia-Neves, Nuno Sousa, Joana A. Palha, and Fernanda Marques. 2015. "From the Periphery to the Brain: Lipocalin-2, a Friend or Foe?” Progress in Neurobiology. https://doi.org/10.1016/j.pneurobio.2015.06.005.

Findley, Caleigh A., Andrzej Bartke, Kevin N. Hascup, and Erin R. Hascup. 2019. "Amyloid BetaRelated Alterations to Glutamate Signaling Dynamics During Alzheimer's Disease Progression." ASN Neuro. https://doi.org/10.1177/1759091419855541.

Fyhn, Marianne, Sturla Molden, Menno P. Witter, Edvard I. Moser, and May-Britt Moser. 2004. "Spatial Representation in the Entorhinal Cortex." Science 305 (5688): 1258-64.

Ghosh, Chaitali, Mohammed Hossain, Jesal Solanki, Aaron Dadas, Nicola Marchi, and Damir Janigro. 2016. "Pathophysiological Implications of Neurovascular P450 in Brain Disorders." Drug Discovery Today. https://doi.org/10.1016/j.drudis.2016.06.004.

Giovanoli, Sandra, Tina Notter, Juliet Richetto, Marie A. Labouesse, Stéphanie Vuillermot, Marco A. 
Riva, and Urs Meyer. 2015. "Late Prenatal Immune Activation Causes Hippocampal Deficits in the Absence of Persistent Inflammation across Aging.” Journal of Neuroinflammation 12 (November): 221.

Götz, Jürgen, Liviu-Gabriel Bodea, and Michel Goedert. 2018. "Rodent Models for Alzheimer Disease." Nature Reviews. Neuroscience 19 (10): 583-98.

Greenspan, P., E. P. Mayer, and S. D. Fowler. 1985. "Nile Red: A Selective Fluorescent Stain for Intracellular Lipid Droplets." The Journal of Cell Biology 100 (3): 965-73.

Hammond, Christine J., Loretta R. Hallock, Raymond J. Howanski, Denah M. Appelt, C. Scott Little, and Brian J. Balin. 2010. "Immunohistological Detection of Chlamydia Pneumoniae in the Alzheimer's Disease Brain.” BMC Neuroscience 11 (September): 121.

Hampel, Harald, Filippo Caraci, A. Claudio Cuello, Giuseppe Caruso, Robert Nisticò, Massimo Corbo, Filippo Baldacci, et al. 2020. "A Path Toward Precision Medicine for Neuroinflammatory Mechanisms in Alzheimer's Disease." Frontiers in Immunology 11 (March): 456.

Han, Xiaojuan, Jialei Zhu, Xinlei Zhang, Qiqi Song, Jianhua Ding, Ming Lu, Sifan Sun, and Gang Hu. 2018. "Plin4-Dependent Lipid Droplets Hamper Neuronal Mitophagy in the MPTP/p-Induced Mouse Model of Parkinson's Disease." Frontiers in Neuroscience. https://doi.org/10.3389/fnins.2018.00397.

Hisanaga, K., S. M. Sagar, K. J. Hicks, R. A. Swanson, and F. R. Sharp. 1990. "C-Fos Proto-Oncogene Expression in Astrocytes Associated with Differentiation or Proliferation but Not Depolarization." Brain Research. Molecular Brain Research 8 (1): 69-75.

Huang, Royston-Luke, Ziqiang Teo, Han Chung Chong, Pengcheng Zhu, Ming Jie Tan, Chek Kun Tan, Chee Ren Ivan Lam, et al. 2011. "ANGPTL4 Modulates Vascular Junction Integrity by Integrin Signaling and Disruption of Intercellular VE-Cadherin and Claudin-5 Clusters." Blood 118 (14): 3990-4002.

Hui, Chin W., Abygaël St-Pierre, Hassan El Hajj, Yvan Remy, Sébastien S. Hébert, Giamal N. Luheshi, Lalit K. Srivastava, and Marie-Ève Tremblay. 2018. "Prenatal Immune Challenge in Mice Leads to Partly Sex-Dependent Behavioral, Microglial, and Molecular Abnormalities Associated with Schizophrenia." Frontiers in Molecular Neuroscience 11 (February): 13.

Hur, Ji-Yeun, Georgia R. Frost, Xianzhong Wu, Christina Crump, Si Jia Pan, Eitan Wong, Marilia Barros, et al. 2020. "The Innate Immunity Protein IFITM3 Modulates $\gamma$-Secretase in Alzheimer's Disease." Nature, September. https://doi.org/10.1038/s41586-020-2681-2.

Jamieson, G. A., N. J. Maitland, G. K. Wilcock, J. Craske, and R. F. Itzhaki. 1991. "Latent Herpes Simplex Virus Type 1 in Normal and Alzheimer's Disease Brains." Journal of Medical Virology 33 (4): 224-27.

Jha, Mithilesh Kumar, Shinrye Lee, Dong Ho Park, Hyun Kook, Keun-Gyu Park, In-Kyu Lee, and Kyoungho Suk. 2015. "Diverse Functional Roles of Lipocalin-2 in the Central Nervous System." Neuroscience and Biobehavioral Reviews 49 (February): 135-56.

Joie, Renaud La, Renaud La Joie, Adrienne V. Visani, Suzanne L. Baker, Jesse A. Brown, Viktoriya Bourakova, Jungho Cha, et al. 2020. "Prospective Longitudinal Atrophy in Alzheimer's Disease Correlates with the Intensity and Topography of Baseline Tau-PET." Science Translational Medicine. https://doi.org/10.1126/scitranslmed.aau5732.

Kang, S. S., Y. Ren, C-C Liu, A. Kurti, K. E. Baker, G. Bu, Y. Asmann, and J. D. Fryer. 2017. "Lipocalin-2 Protects the Brain during Inflammatory Conditions." Molecular Psychiatry 23 (2): 344-50.

Kim, Eun Kyung, and Eui-Ju Choi. 2010. "Pathological Roles of MAPK Signaling Pathways in Human Diseases." Biochimica et Biophysica Acta 1802 (4): 396-405.

Kim, Jae-Hong, Pan-Woo Ko, Ho-Won Lee, Ji-Young Jeong, Maan-Gee Lee, Jong-Heon Kim, Won-Ha Lee, Ri Yu, Won-Jong Oh, and Kyoungho Suk. 2017. "Astrocyte-Derived Lipocalin-2 Mediates Hippocampal Damage and Cognitive Deficits in Experimental Models of Vascular Dementia." Glia 65 (9): 1471-90.

Kinney, Jefferson W., Shane M. Bemiller, Andrew S. Murtishaw, Amanda M. Leisgang, Arnold M. 
Salazar, and Bruce T. Lamb. 2018. "Inflammation as a Central Mechanism in Alzheimer's Disease." Alzheimer's \& Dementia : Translational Research \& Clinical Interventions 4: 575.

Kitazawa, Masashi, Salvatore Oddo, Tritia R. Yamasaki, Kim N. Green, and Frank M. LaFerla. 2005. "Lipopolysaccharide-Induced Inflammation Exacerbates Tau Pathology by a Cyclin-Dependent Kinase 5-Mediated Pathway in a Transgenic Model of Alzheimer's Disease." The Journal of Neuroscience: The Official Journal of the Society for Neuroscience 25 (39): 8843-53.

Knuesel, Irene, Laurie Chicha, Markus Britschgi, Scott A. Schobel, Michael Bodmer, Jessica A. Hellings, Stephen Toovey, and Eric P. Prinssen. 2014. "Maternal Immune Activation and Abnormal Brain Development across CNS Disorders." Nature Reviews. Neurology 10 (11): 643-60.

Koopmans, Frank, Pim van Nierop, Maria Andres-Alonso, Andrea Byrnes, Tony Cijsouw, Marcelo P. Coba, L. Niels Cornelisse, et al. 2019. "SynGO: An Evidence-Based, Expert-Curated Knowledge Base for the Synapse." Neuron 103 (2): 217-34.e4.

Kövari, E., F. R. Herrmann, P. R. Hof, and C. Bouras. 2013. "The Relationship between Cerebral Amyloid Angiopathy and Cortical Microinfarcts in Brain Ageing and Alzheimer's Disease." Neuropathology and Applied Neurobiology 39 (5): 498-509.

Krstic, Dimitrije, Amrita Madhusudan, Jana Doehner, Prisca Vogel, Tina Notter, Claudine Imhof, Abigail Manalastas, et al. 2012. "Systemic Immune Challenges Trigger and Drive Alzheimer-like Neuropathology in Mice." Journal of Neuroinflammation 9: 151.

Leung, Rufina, Petroula Proitsi, Andrew Simmons, Katie Lunnon, Andreas Güntert, Deborah Kronenberg, Megan Pritchard, et al. 2013. "Inflammatory Proteins in Plasma Are Associated with Severity of Alzheimer's Disease." PloS One 8 (6): e64971.

Licastro, Federico, Luigi Maria Edoardo Grimaldi, Massimiliano Bonafè, Chiappelli Martina, Fabiola Olivieri, Luca Cavallone, Simona Giovanietti, Eliezer Masliah, and Claudio Franceschi. 2003. "Interleukin-6 Gene Alleles Affect the Risk of Alzheimer's Disease and Levels of the Cytokine in Blood and Brain." Neurobiology of Aging 24 (7): 921-26.

Ling, E. A., and W. C. Wong. 1993. "The Origin and Nature of Ramified and Amoeboid Microglia: A Historical Review and Current Concepts." Glia 7 (1): 9-18.

Lin, Yen-Feng, Albert Vernon Smith, Thor Aspelund, Rebecca A. Betensky, Jordan W. Smoller, Vilmundur Gudnason, Lenore J. Launer, and Deborah Blacker. 2019. "Genetic Overlap between Vascular Pathologies and Alzheimer's Dementia and Potential Causal Mechanisms." Alzheimer's \& Dementia: The Journal of the Alzheimer's Association 15 (1): 65-75.

Llorens, Franc, Peter Hermann, Anna Villar-Piqué, Daniela Diaz-Lucena, Katarina Nägga, Oskar Hansson, Isabel Santana, et al. 2020a. "Cerebrospinal Fluid Lipocalin 2 as a Novel Biomarker for the Differential Diagnosis of Vascular Dementia." Nature Communications 11 (1): 619.

- 2020b. "Cerebrospinal Fluid Lipocalin 2 as a Novel Biomarker for the Differential Diagnosis of Vascular Dementia." Nature Communications 11 (1): 619.

Masters, Colin L., Randall Bateman, Kaj Blennow, Christopher C. Rowe, Reisa A. Sperling, and Jeffrey L. Cummings. 2015. “Alzheimer's Disease.” Nature Reviews Disease Primers. https://doi.org/10.1038/nrdp.2015.56.

Masuda, Takahiro, Roman Sankowski, Ori Staszewski, Chotima Böttcher, Lukas Amann, Sagar, Christian Scheiwe, et al. 2019. "Spatial and Temporal Heterogeneity of Mouse and Human Microglia at Single-Cell Resolution.” Nature 566 (7744): 388-92.

McHugh, Stephen B., Burkhard Niewoehner, J. N. P. Rawlins, and David M. Bannerman. 2008. "Dorsal Hippocampal N-Methyl-D-Aspartate Receptors Underlie Spatial Working Memory Performance during Non-Matching to Place Testing on the T-Maze." Behavioural Brain Research 186 (1): 41-47.

Metti, Andrea L., and Jane A. Cauley. 2012. "How Predictive of Dementia Are Peripheral Inflammatory Markers in the Elderly?" Neurodegenerative Disease Management 2 (6): 609-22.

Miedel, Christian J., Jennifer M. Patton, Andrew N. Miedel, Edward S. Miedel, and Jonathan M. Levenson. 2017. "Assessment of Spontaneous Alternation, Novel Object Recognition and Limb Clasping in Transgenic Mouse Models of Amyloid- $\beta$ and Tau Neuropathology." Journal of Visualized Experiments: JoVE, no. 123 (May). https://doi.org/10.3791/55523. 
Miklossy, Judith, Kamel Khalili, Lise Gern, Rebecca L. Ericson, Pushpa Darekar, Lorie Bolle, Jean Hurlimann, and Bruce J. Paster. 2004. "Borrelia Burgdorferi Persists in the Brain in Chronic Lyme Neuroborreliosis and May Be Associated with Alzheimer Disease." Journal of Alzheimer's Disease: JAD 6 (6): 639-49; discussion 673-81.

Morgan, Angharad R., Samuel Touchard, Claire Leckey, Caroline O’Hagan, Alejo J. Nevado-Holgado, NIMA Consortium, Frederik Barkhof, et al. 2019. "Inflammatory Biomarkers in Alzheimer's Disease Plasma." Alzheimer's \& Dementia: The Journal of the Alzheimer's Association 15 (6): 77687.

Naudé, Petrus J. W., Csaba Nyakas, Lee E. Eiden, Djida Ait-Ali, Ragna van der Heide, Sebastiaan Engelborghs, Paul G. M. Luiten, Peter P. De Deyn, Johan A. den Boer, and Ulrich L. M. Eisel. 2012. "Lipocalin 2: Novel Component of Proinflammatory Signaling in Alzheimer's Disease." The FASEB Journal 26 (7): 2811.

Newcombe, Estella A., Judith Camats-Perna, Mallone L. Silva, Nicholas Valmas, Tee Jong Huat, and Rodrigo Medeiros. 2018. "Inflammation: The Link between Comorbidities, Genetics, and Alzheimer's Disease.” Journal of Neuroinflammation 15 (1): 276.

Palenski, Tammy L., Christine M. Sorenson, Colin R. Jefcoate, and Nader Sheibani. 2013. "Lack of Cyp1b1 Promotes the Proliferative and Migratory Phenotype of Perivascular Supporting Cells." Laboratory Investigation; a Journal of Technical Methods and Pathology 93 (6): 646-62.

Palop, Jorge J., and Lennart Mucke. 2010. “Amyloid- $\beta$-induced Neuronal Dysfunction in Alzheimer's Disease: From Synapses toward Neural Networks." Nature Neuroscience 13 (7): 812-18.

Park, Laibaik, Karin Hochrainer, Yorito Hattori, Sung Ji Ahn, Antoine Anfray, Gang Wang, Ken Uekawa, et al. 2020. "Tau Induces PSD95-Neuronal NOS Uncoupling and Neurovascular Dysfunction Independent of Neurodegeneration.” Nature Neuroscience 23 (9): 1079-89.

Patrich, Eti, Yael Piontkewitz, Asher Peretz, Ina Weiner, and Bernard Attali. 2016. "Maternal Immune Activation Produces Neonatal Excitability Defects in Offspring Hippocampal Neurons from Pregnant Rats Treated with Poly I:C.” Scientific Reports 6 (January): 19106.

Perry, V. Hugh, Colm Cunningham, and Clive Holmes. 2007. "Systemic Infections and Inflammation Affect Chronic Neurodegeneration." Nature Reviews. Immunology 7 (2): 161-67.

Pioli, Elsa Y., Brianna N. Gaskill, Gary Gilmour, Mark D. Tricklebank, Sophie L. Dix, David Bannerman, and Joseph P. Garner. 2014. "An Automated Maze Task for Assessing HippocampusSensitive Memory in Mice.” Behavioural Brain Research 261 (100): 249.

Price, Brittani R., Christopher M. Norris, Pradoldej Sompol, and Donna M. Wilcock. 2018. “An Emerging Role of Astrocytes in Vascular Contributions to Cognitive Impairment and Dementia." Journal of Neurochemistry 144 (5): 644-50.

Qiu, Wei Qiao, and Marshal F. Folstein. 2006. "Insulin, Insulin-Degrading Enzyme and Amyloid-Beta Peptide in Alzheimer's Disease: Review and Hypothesis." Neurobiology of Aging 27 (2): 190-98.

Quintanilla. 2004. "Interleukin-6 Induces Alzheimer-Type Phosphorylation of Tau Protein by Deregulating the cdk5/p35 Pathway." Experimental Cell Research 295 (1): 245-57.

Rahimi, Jasmin, and Gabor G. Kovacs. 2014. "Prevalence of Mixed Pathologies in the Aging Brain." Alzheimer's Research \& Therapy 6 (9): 82.

Readhead, Ben, Jean-Vianney Haure-Mirande, Cory C. Funk, Matthew A. Richards, Paul Shannon, Vahram Haroutunian, Mary Sano, et al. 2018. "Multiscale Analysis of Independent Alzheimer's Cohorts Finds Disruption of Molecular, Genetic, and Clinical Networks by Human Herpesvirus." Neuron 99 (1): 64-82.e7.

Rice, Rachel A., Nicole C. Berchtold, Carl W. Cotman, and Kim N. Green. 2014. "Age-Related Downregulation of the CaV3.1 T-Type Calcium Channel as a Mediator of Amyloid Beta Production." Neurobiology of Aging 35 (5): 1002-11.

Saeed, Sadia, Jessica Quintin, Hindrik H. D. Kerstens, Nagesha A. Rao, Ali Aghajanirefah, Filomena Matarese, Shih-Chin Cheng, et al. 2014. "Epigenetic Programming of Monocyte-to-Macrophage Differentiation and Trained Innate Immunity." Science 345 (6204): 1251086.

Sangwung, Panjamaporn, Guangjin Zhou, Lalitha Nayak, E. Ricky Chan, Sandeep Kumar, Dong-Won 
Kang, Rongli Zhang, et al. 2017. "KLF2 and KLF4 Control Endothelial Identity and Vascular Integrity." JCI Insight 2 (4): e91700.

Santos, Cláudia Y., Peter J. Snyder, Wen-chih Wu, Mia Zhang, Ana Echeverria, and Jessica Alber. 2017. "Pathophysiologic Relationship between Alzheimer's Disease, Cerebrovascular Disease, and Cardiovascular Risk: A Review and Synthesis." Alzheimer's \& Dementia: Diagnosis, Assessment \& Disease Monitoring. https://doi.org/10.1016/j.dadm.2017.01.005.

Sasaki-Hamada, Sachie, Masaatsu Ikeda, and Jun-Ichiro Oka. 2019. "Glucagon-like Peptide-2 Rescues Memory Impairments and Neuropathological Changes in a Mouse Model of Dementia Induced by the Intracerebroventricular Administration of Streptozotocin." Scientific Reports. https://doi.org/10.1038/s41598-019-50167-3.

Serrano-Pozo, Alberto, Matthew L. Mielke, Teresa Gómez-Isla, Rebecca A. Betensky, John H. Growdon, Matthew P. Frosch, and Bradley T. Hyman. 2011. "Reactive Glia Not Only Associates with Plaques but Also Parallels Tangles in Alzheimer's Disease." The American Journal of Pathology 179 (3): 1373-84.

Siemsen, B. M., J. D. Landin, J. A. McFaddin, K. N. Hooker, L. J. Chandler, and M. D. Scofield. 2020. "Chronic Intermittent Ethanol and Lipopolysaccharide Exposure Differentially Alter Iba-1-Derived Microglia Morphology in the Prelimbic Cortex and Nucleus Accumbens Core." bioRxiv. https://doi.org/10.1101/566034.

Silveira, Vivian T., Daniel Castro Medeiros, Jivago Ropke, Patricia A. Guidine, Gustavo H. Rezende, Marcio Flavio D. Moraes, Eduardo Mazoni A, Danielle Macedo, Fabricio A. Moreira, and Antonio Carlos P. Oliveira. 2017. "Effects of Early or Late Prenatal Immune Activation in Mice on Behavioral and Neuroanatomical Abnormalities Relevant to Schizophrenia in the Adulthood." International Journal of Developmental Neuroscience. https://doi.org/10.1016/j.ijdevneu.2017.01.009.

Song, Juhyun, and Oh Yoen Kim. 2018. "Perspectives in Lipocalin-2: Emerging Biomarker for Medical Diagnosis and Prognosis for Alzheimer's Disease." Clinical Nutrition Research. https://doi.org/10.7762/cnr.2018.7.1.1.

Stozická, Z., N. Zilka, and M. Novák. 2007. "Risk and Protective Factors for Sporadic Alzheimer's Disease." Acta Virologica 51 (4): 205-22.

Streit, Wolfgang J., Sharon A. Walter, and Nathan A. Pennell. 1999. "Reactive Microgliosis." Progress in Neurobiology. https://doi.org/10.1016/s0301-0082(98)00069-0.

Thal, Dietmar Rudolf, Estifanos Ghebremedhin, Udo Rüb, Haruyasu Yamaguchi, Kelly Del Tredici, and Heiko Braak. 2002. "Two Types of Sporadic Cerebral Amyloid Angiopathy." Journal of Neuropathology and Experimental Neurology 61 (3): 282-93.

Toledo, Jon B., Steven E. Arnold, Kevin Raible, Johannes Brettschneider, Sharon X. Xie, Murray Grossman, Sarah E. Monsell, Walter A. Kukull, and John Q. Trojanowski. 2013. "Contribution of Cerebrovascular Disease in Autopsy Confirmed Neurodegenerative Disease Cases in the National Alzheimer's Coordinating Centre." Brain. https://doi.org/10.1093/brain/awt188.

Town, Terrence, David Jeng, Lena Alexopoulou, Jun Tan, and Richard A. Flavell. 2006. "Microglia Recognize Double-Stranded RNA via TLR3.” Journal of Immunology 176 (6): 3804-12.

Van Hove, Hannah, Liesbet Martens, Isabelle Scheyltjens, Karen De Vlaminck, Ana Rita Pombo Antunes, Sofie De Prijck, Niels Vandamme, et al. 2019. "A Single-Cell Atlas of Mouse Brain Macrophages Reveals Unique Transcriptional Identities Shaped by Ontogeny and Tissue Environment." Nature Neuroscience 22 (6): 1021-35.

Walkera, Frederick Rohan, Michael Nilsson, and Kimberley Jones. 2013. "Acute and Chronic StressInduced Disturbances of Microglial Plasticity, Phenotype and Function." Current Drug Targets 14 (October): 1262.

Walker, F. Rohan, Sarah B. Beynon, Kimberley A. Jones, Zidan Zhao, Ratchaniporn Kongsui, Murray Cairns, and Michael Nilsson. 2014. "Dynamic Structural Remodelling of Microglia in Health and Disease: A Review of the Models, the Signals and the Mechanisms." Brain, Behavior, and Immunity 37 (March): 1-14. 
Wallenius, Ville, Kristina Wallenius, Bo Ahrén, Mats Rudling, Hans Carlsten, Suzanne L. Dickson, Claes Ohlsson, and John-Olov Jansson. 2002. "Interleukin-6-Deficient Mice Develop Mature-Onset Obesity." Nature Medicine 8 (1): 75-79.

Wang, Feixue, Yu Cao, Lina Ma, Hui Pei, Wolf Dieter Rausch, and Hao Li. 2018. "Dysfunction of Cerebrovascular Endothelial Cells: Prelude to Vascular Dementia." Frontiers in Aging Neuroscience 10 (November): 376.

Williams, J., M. Dragunow, P. Lawlor, S. Mason, W. C. Abraham, J. Leah, R. Bravo, J. Demmer, and W. Tate. 1995. "Krox20 May Play a Key Role in the Stabilization of Long-Term Potentiation." Brain Research. Molecular Brain Research 28 (1): 87-93.

Wischhof, Lena, Ellen Irrsack, Carmen Osorio, and Michael Koch. 2015. "Prenatal LPS-Exposure--a Neurodevelopmental Rat Model of Schizophrenia--Differentially Affects Cognitive Functions, Myelination and Parvalbumin Expression in Male and Female Offspring." Progress in NeuroPsychopharmacology \& Biological Psychiatry 57 (March): 17-30.

Xie, Yan-Chun, Zhao-Hui Yao, Xiao-Li Yao, Jian-Zhen Pan, Shao-Feng Zhang, Yong Zhang, and JiChang Hu. 2018. "Glucagon-Like Peptide-2 Receptor Is Involved in Spatial Cognitive Dysfunction in Rats After Chronic Cerebral Hypoperfusion." Journal of Alzheimer's Disease: JAD 66 (4): 155976.

Yin, Jie, Katherine L. Valin, Michael L. Dixon, and Jianmei W. Leavenworth. 2017. "The Role of Microglia and Macrophages in CNS Homeostasis, Autoimmunity, and Cancer." Journal of Immunology Research 2017 (December). https://doi.org/10.1155/2017/5150678.

Young, Kimberly, and Helena Morrison. 2018. "Quantifying Microglia Morphology from Photomicrographs of Immunohistochemistry Prepared Tissue Using ImageJ." Journal of Visualized Experiments: JoVE, no. 136 (June). https://doi.org/10.3791/57648. 
A $\quad$ Figure 1

Polyl:Ci.v. Polyl:Ci.p.

$5 \mathrm{mg} / \mathrm{Kg} \quad 20 \mathrm{mg} / \mathrm{Kg}$

$\begin{array}{cccccc}V & B D & V_{3} \text { mo } & 6 \text { mo } & 9 \text { mo } & 16 \text { mo } \\ \text { GD17 } & \mathbf{l} & \mathbf{I} & \mathbf{I} & \mathbf{I} & \mathbf{I}\end{array}$

\section{Behavior}

Transcriptomics

Biochemistry

Histology

$\begin{array}{lll}x & x & x \\ x & x & x \\ x & x & x \\ x & x & x\end{array}$

C

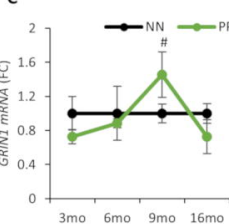

D

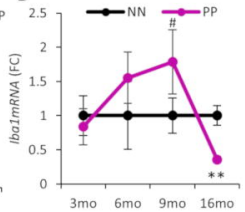

E

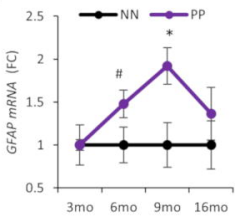

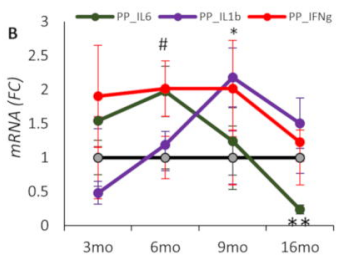

F

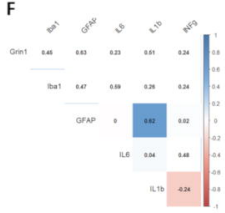




\section{Figure 2}

A

\begin{tabular}{|c|c|c|}
\hline & & \\
\hline & NN & PP \\
\hline pTau & 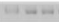 & $-6=62$ \\
\hline Tau & 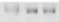 & ேே06 \\
\hline GFAP & $--\cdots$ & ---- \\
\hline
\end{tabular}

Synaptophysin - -

$\beta$-actin - $-\ldots-\ldots-\cdots$ 9 months

\section{NN}

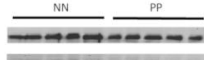

Tau

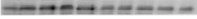

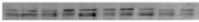

GFAP

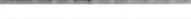

Synaptophysin

$\beta$-actin

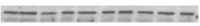

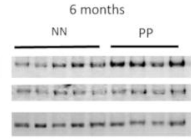

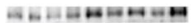

드. - - - 16 months
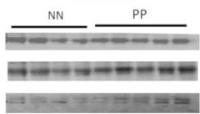

$-\cdots+\cdots+\cdots$ $\rightarrow-\cdots-\cdots$

\section{B}

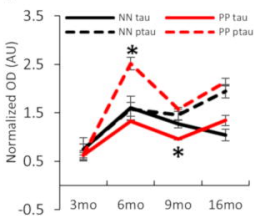

D

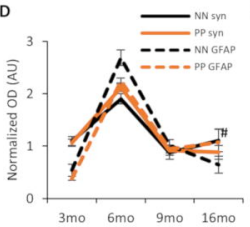

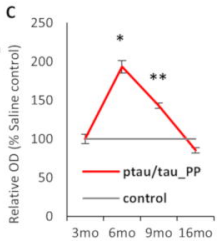

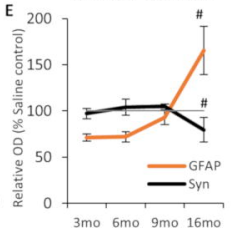

F 9mo NN

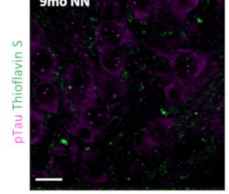

\section{$9 \mathrm{mo}$ PP}

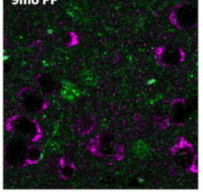

G

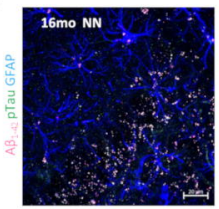

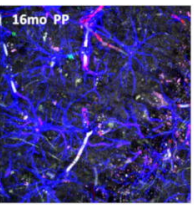


A
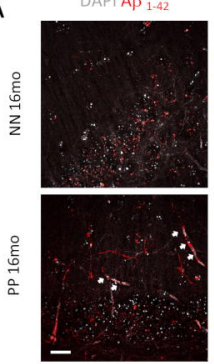

*

D

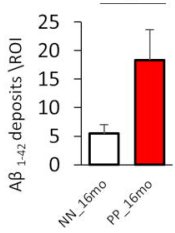

DAPI GFAP A $\beta_{1-42}$
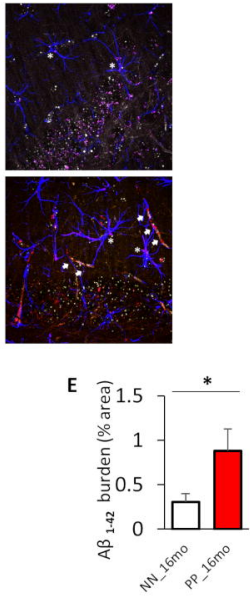

B

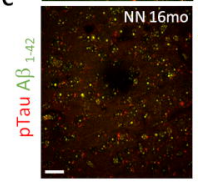

F
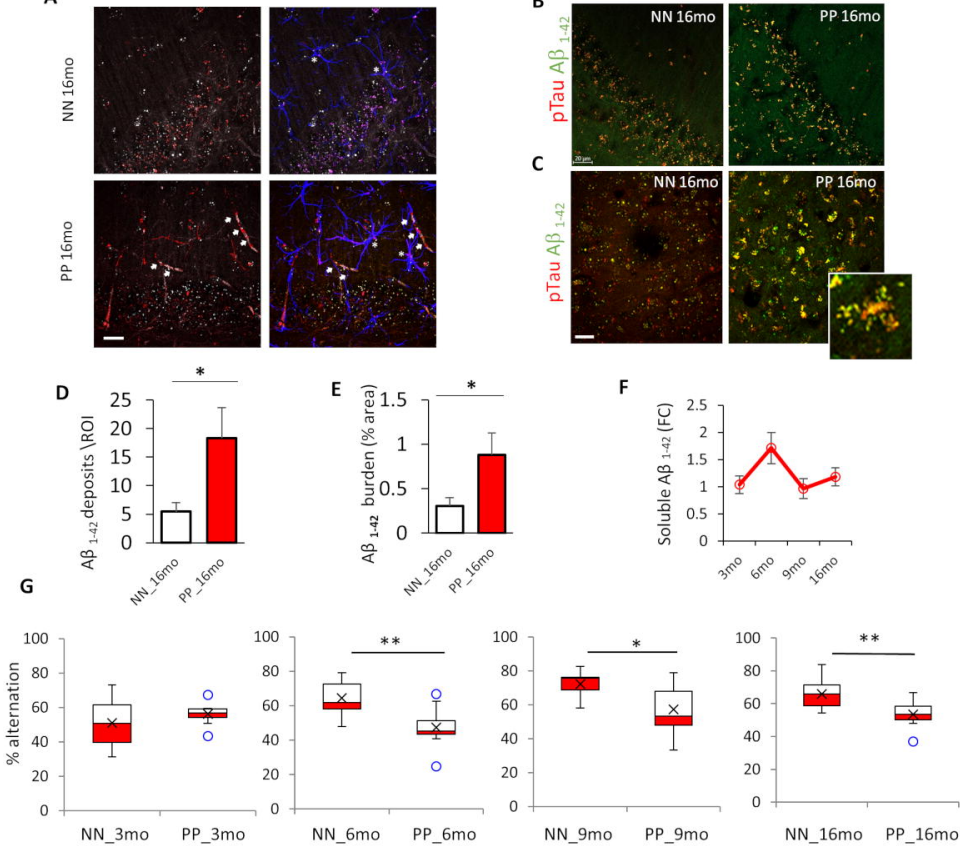
Figure 5
A

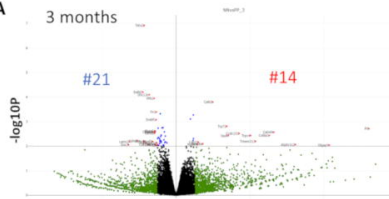

$\log 2$ Foldcha

C 9 months-

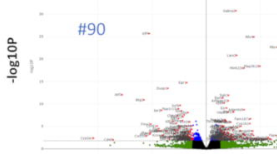

log2foldchange
B 6 months

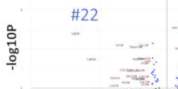

\#10

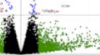

$\log 2$ Foldchange

D 16 months

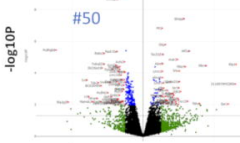

log2foldchange
E
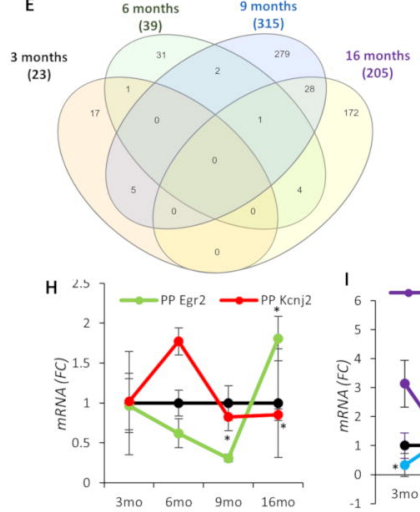

\#106

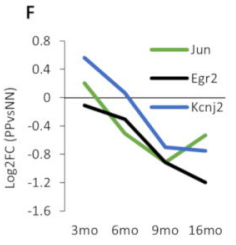

G

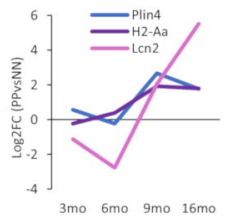

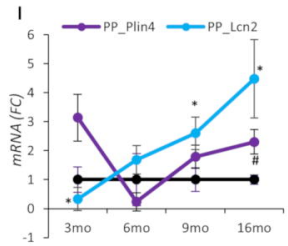

J

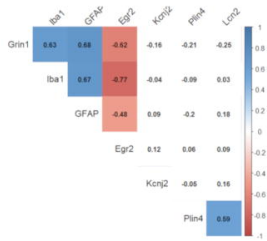


A $\quad$ Figure 6

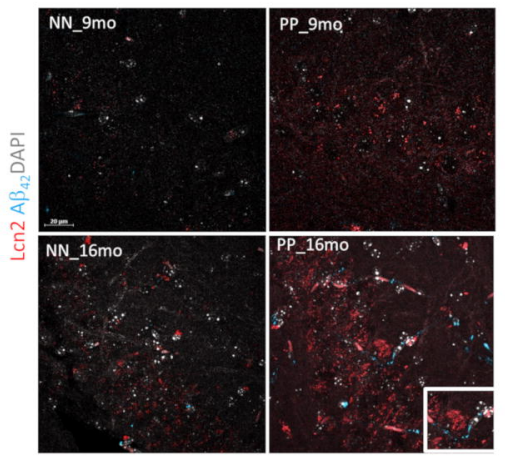

C

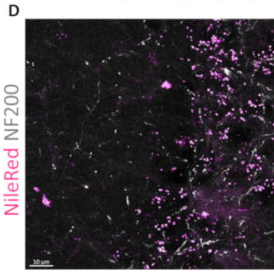

NN_9mo

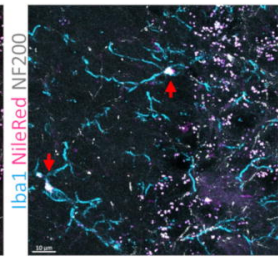

B
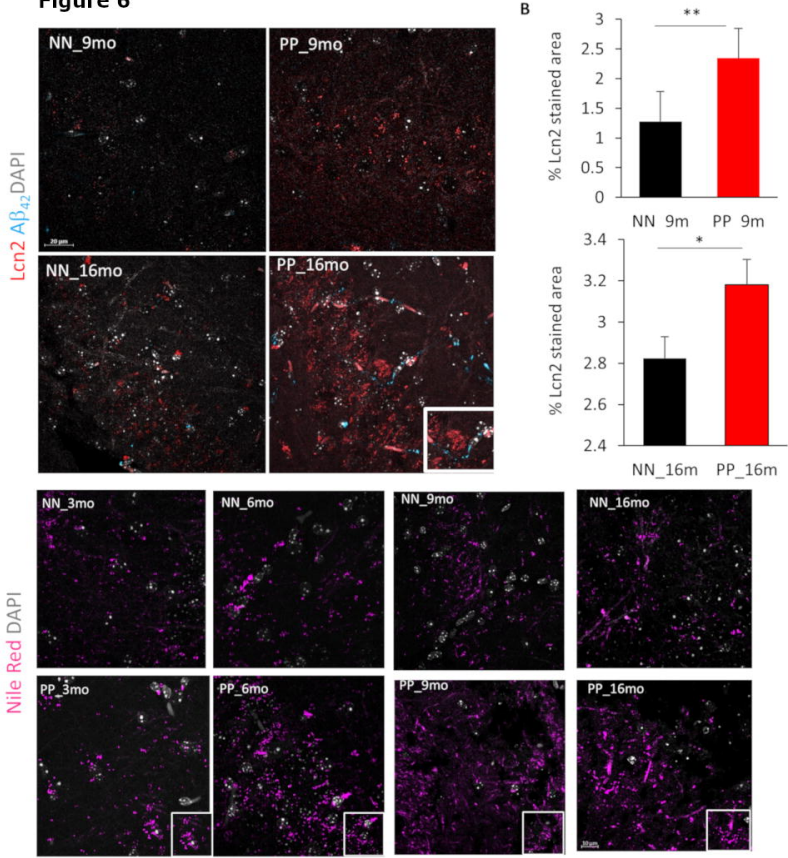
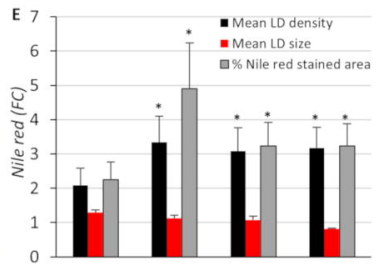

PP 3 mo PP $6 \mathrm{mo}$ PP $9 \mathrm{mo}$ PP $16 \mathrm{mo}$ 

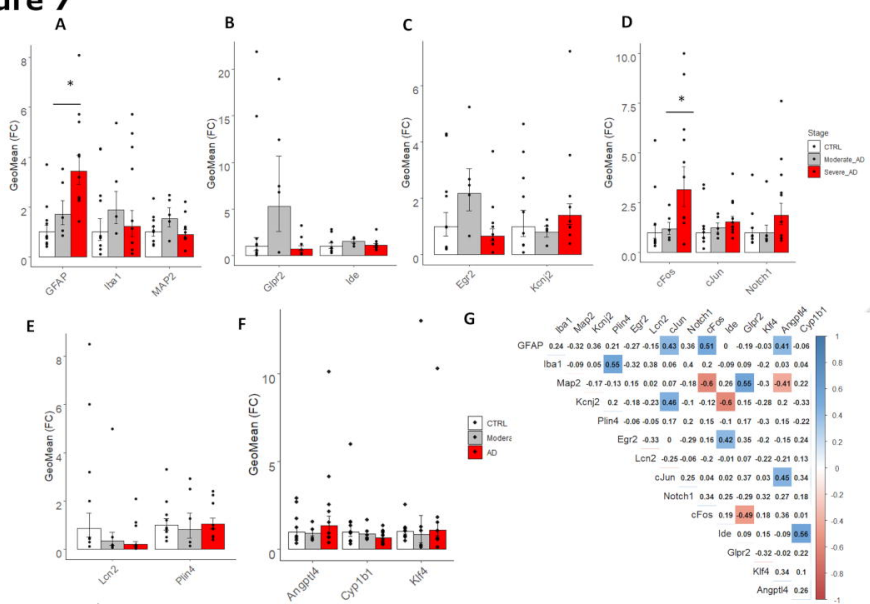

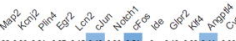
GFAP $0.24-0.320 .560 .21-9.27-6.150 .430 .350 .510 .0 .19-0.530 .41 .0 .06$

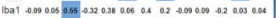
Map2 $0.17-0.130 .150 .220 .07-0.10-0.60 .260 .55-0.3-9.410 .22$

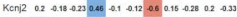

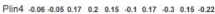
Egr2 -0.53 0 - $0.290 .160 .420 .35-0.2-0.150 .24$ Lon2 $0.26-0.06 \quad 0.2-0.010 .07-0.22-9.210 .13$ cJun $\$ .25$ e.94 $0.020 .370 .03 \quad 0.260 .34$ Noich $10.34 \quad 0.25 \quad 0.290 .32 \quad 0.27 \quad 0.18$ CFOS 0.190 .490 .180 .300 .01 Ide $0.090 .15-9.090 .66$ Glpr2 $-0.32-0.020 .22$ KIf4 0.34 a.t Angpt/4 0.26
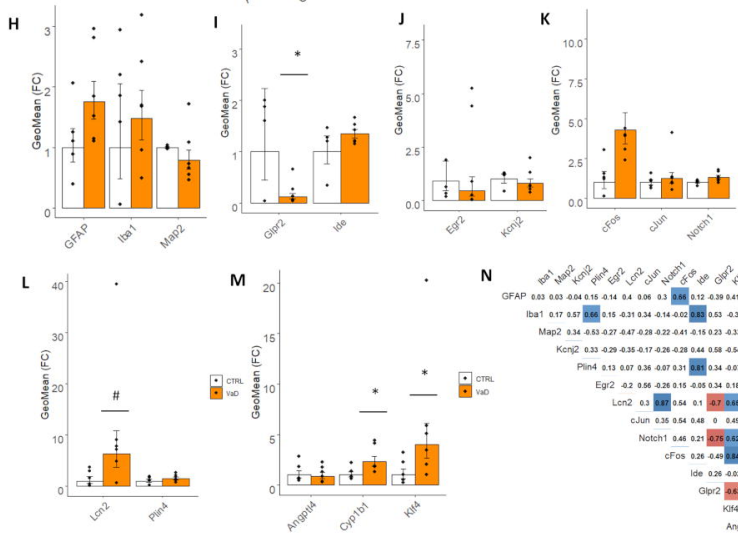

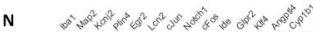

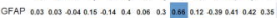

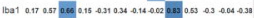

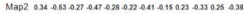

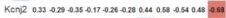
Pin4 0.13 0.07 0.36 -0.070.31 0.01 0.34 -0.07 0.15 -0.19 Egr2 $-0.20 .56-0.260 .15-0.050 .340 .18065-0.03$

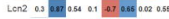
cJun 0.250 .540 .4800 .490 .40 .12 Noteh1 $0.450 .2107075062-0140.62$ efos 0.260 .490 .640 .060 .64 Ide $0.26+0.020 .13+0.17$ Glpr2 -0.69 $0.12-0.02$ K114.021 070 Angpt/4 -0.20 